Research Articles: Cellular/Molecular

\title{
Chemical signaling regulates axon regeneration via the GPCR-Gqa pathway in Caenorhabditis elegans
}

https://doi.org/10.1523/JNEUROSCI.0929-21.2021

Cite as: J. Neurosci 2021; 10.1523/JNEUROSCI.0929-21.2021

Received: 2 May 2021

Revised: 4 October 2021

Accepted: 23 November 2021

This Early Release article has been peer-reviewed and accepted, but has not been through the composition and copyediting processes. The final version may differ slightly in style or formatting and will contain links to any extended data.

Alerts: Sign up at www.jneurosci.org/alerts to receive customized email alerts when the fully formatted version of this article is published.

Copyright (C) 2021 Shimizu et al.

This is an open-access article distributed under the terms of the Creative Commons Attribution 4.0 International license, which permits unrestricted use, distribution and reproduction in any medium provided that the original work is properly attributed. 


\section{Chemical signaling regulates axon regeneration 2 via the GPCR-Gq $\alpha$ pathway in Caenorhabditis 3 elegans}

4

6

7

\section{Running Title: Chemical signaling in axon regeneration}

Tatsuhiro Shimizu1, ${ }^{\star}$, Kayoko Sugiura $1{ }^{,}$, , Yoshiki Sakai1, Abdul R. Dar2, Rebecca A. Butcher2, Kunihiro Matsumoto1, and Naoki Hisamoto1

1Division of Biological Science, Graduate School of Science, Nagoya University, Chikusa-ku, Nagoya 464-8602, Japan

2Department of Chemistry, University of Florida, Gainesville, FL, USA

*These authors contributed equally to this work.

To whom correspondence should be addressed:

Kunihiro Matsumoto and Naoki Hisamoto

Division of Biological Science, Graduate School of Science, Nagoya University, Chikusa-ku, Nagoya 464-8602, Japan.

E-mail address: g44177a@nucc.cc.nagoya-u.ac.jp (K. M.) i45556a@cc.nagoya-u.ac.jp (N. H.)

T.S., Y.S., N.H., and K.M. designed the experiments and analyzed data. T.S., K.S., and Y.S. performed genetic and biological experiments. R.A.B. and A.R.D. produced ascr\#5. T.S. and Y.S. performed all of the other experiments. K.M. and N.H. wrote the manuscript. 
Shimizu et al., 2

Number of Pages: 36 pages, without figures and tables

Number of Figures and Tables: 9 figures and 2 tables

Number of words for Abstract, Introduction and Discussion: 135, 644, and 1031

words, respectively.

\section{Acknowledgements}

We thank Dr. Strahil Iv. Pastuhov, Dr. Cori Bargmann, Caenorhabditis Genetic

Center (CGC), National Bio-Resource Project and C. elegans Knockout

Consortium for technical assistance and materials. Some strains were provided by the CGC, which is funded by NIH Office of Research Infrastructure Programs (P40 OD10440). This work was supported by grants from the Ministry of Education, Culture and Science of Japan (K.M.), the Project for Elucidating and Controlling Mechanisms of Aging, Longevity from Japan Agency for Medical Research and Development, AMED, under Grant Number JP21gm5010001 (to N.H.), NSF CHE-1555050 (to R.A.B.), and NIH R01GM118775 (to R.A.B.). T.S. and Y.S. were supported by a Japan Society for the Promotion of Science Research Fellowship.

The authors declare that they have no conflict of interest. 


\section{Abstract}

Chemical communication controls a wide range of behaviors via conserved signaling networks. Axon regeneration in response to injury is determined by the interaction between the extracellular environment and intrinsic growth potential. In this study, we investigated the role of chemical signaling in axon regeneration in Caenorhabditis elegans. We find that the enzymes involved in ascaroside pheromone biosynthesis, ACOX-1.1, ACOX-1.2, and DAF-22, participate in axon regeneration by producing a dauer-inducing ascaroside, ascr\#5. We demonstrate that the chemoreceptor genes, srg-36 and srg-37, which encode G protein-coupled receptors (GPCRs) for ascr\#5, are required for adult-specific axon regeneration. Furthermore, the activating mutation in egl-30 encoding Gq $\alpha$ suppresses axon regeneration defective phenotype in acox-1.1 and srg-36 srg-37 mutants. Therefore, the ascaroside signaling system provides a unique example of a signaling molecule that regulates the regenerative pathway in the nervous system. 
Significance Statement

In C. elegans, axon regeneration is positively regulated by the EGL-30

Gq $\alpha-J N K$ MAP kinase cascade. However, it remains unclear what signals activate the EGL-30 pathway in axon regeneration. Here, we show that SRG-36 and SRG-37 act as upstream GPCRs that activate EGL-30. C. elegans secretes a family of small-molecule pheromones called ascarosides, which serve various functions in chemical signaling. SRG-36 and SRG-37 are GPCRs for the dauer-inducing ascaroside, ascr\#5.

Consistent with this, we found that ascr\#5 activates the axon regeneration pathway via SRG-36/SRG-37 and EGL-30. Thus, ascaroside signaling promotes axon regeneration by activating the GPCR-Gq $\alpha$ pathway. 
Shimizu et al., 5

\section{Introduction}

The ability of axons to regenerate after an injury is a fundamental and conserved property of neurons, which is influenced by the balance between extrinsic factors that inhibit or promote axon outgrowth and intrinsic growth capacity of neurons (Kaplan et al., 2015). In adult mammals, regeneration following nerve injury occurs efficiently in the peripheral nervous system, whereas the central nervous system does not successfully regenerate after injury (Mahar and Cavalli, 2018). This difference in regeneration potential has been attributed to the combined effects of extrinsic signals and intrinsic growth capacity (Tedeshi and Bradke, 2017). However, the mechanisms underlying the regulation of regeneration by the extracellular environment in the adult nervous system remain unclear.

Recent studies on axon regeneration in the genetic model animal, Caenorhabditis elegans, have revealed that the JNK MAP kinase (MAPK) cascade is a key intrinsic regulator of axon regeneration and may act to sense axonal damage (Fig. 1A; Hisamoto and Matsumoto, 2017). The JNK pathway consists of MLK-1 MAPKKK, MEK-1 MAPKK, and KGB-1 JNK; it is inactivated at the KGB-1 activation step by VHP-1, a member of the MAPK phosphatase family (Mizuno et al., 2004). The vhp-1 loss-of-function mutation leads to hyperactivation of the JNK pathway, resulting in developmental arrest at an early larval stage. We had previously conducted a genome-wide RNAi screening for suppressors of vhp-1 lethality and isolated 92 svh genes (Li et al., 2012; Shimizu et al., 2021). Analysis of these svh genes shed new light on the regulation of axon regeneration. Moreover, two distinct protein kinases act as MAP4Ks for MLK-1 MAPKKK in a life stage-dependent manner (Pastuhov et al., 2012). Ste20-related kinase, MAX-2, phosphorylates and activates MLK-1 primarily at the L4 stage to promote axon regeneration. In contrast, the protein kinase C 
Shimizu et al., 6

(PKC) homolog, TPA-1, can activate MLK-1 at the young adult stage but not at the L4 stage. The Gq $\alpha$ protein, EGL-30, acts as an upstream component of TPA-1. EGL-30 activates a phospholipase $C \beta$ (PLC $\beta), E G L-8$, which in turn generates diacyl-glycerol (DAG), an activator of TPA-1 (Fig. 1A). Endocannabinoid anandamide (AEA) inhibits axon regeneration via the Go $\alpha$ protein, GOA-1, which antagonizes EGL-30. NPR-19 and NPR-32 function as G protein-coupled receptors (GPCRs) for AEA (Pastuhov et al., 2012). Therefore, increased signaling from the AEA pathway suppresses the EGL-30-EGL-8TPA-1 signaling cascade, which inhibits the activation of the JNK pathway, thereby reducing axon regeneration. However, it remains unclear what signals activate the EGL-30 pathway in the regulation of axon regeneration.

In many organisms, the extracellular environment is interpreted through chemical signaling systems mediated by small molecules (Peso et al., 2015). C. elegans secretes a family of small-molecule pheromones called ascarosides, which participate in diverse chemical signaling functions (Park et al., 2019). In particular, ascarosides facilitate the transition to the non-feeding, long-lived, and highly stress-tolerant dauer stage (Butcher et al., 2007). Ascarosides also have a number of other important effects on behaviors, such as aggregation, avoidance, and mating attraction. Therefore, the animals optimize the extent of these developmental or behavioral decisions by assessing the environmental conditions that affect their survival strategies (Butcher, 2017). Ascarosides are initially synthesized as glycolipids with extremely long side chains that are subsequently shortened by the peroxisomal fatty acid (FA) $\beta$-oxidation pathway (Butcher, 2017). This pathway is composed of four enzymes: acyl-CoA oxidases (ACOXs), enoyl-CoA hydratase (MAOC-1), hydroxyacyl-CoA dehydrogenase (DHS-28), and $\beta$-ketoacyl-CoA thiolase (DAF-22). More than 200 ascaroside-like 
130 compounds have been identified by metabolomics and they are divided into two 131 main classes: $\omega$-ascarosides and ( $\omega$-1)-ascarosides (von Reuss et al., 2017).

132 In this study, we found that one dauer-inducing $\omega$-ascaroside, ascr\#5

133 (asc- $\omega \mathrm{C} 3$; C3), acts on the EGL-30 Gq signaling pathway to promote axon

134 regeneration. Furthermore, we revealed that the srg-36 and srg-37genes, which

135 encode GPCRs for ascr\#5, are required for axon regeneration by functioning

136 upstream of EGL-30. These findings strengthen the link between chemical

137 inputs and a conserved regulatory mechanism for axon regeneration. 
Shimizu et al., 8

140

141

\section{Materials and Methods}

\section{C. elegans strains}

The $C$. elegans strains used in this study are listed in Table 1. The strains, KU501, KU456, and KU457 have been previously reported (Pastuhov et al., 2012). All strains were maintained on nematode growth medium plates and fed with bacteria of the OP50 strain of Escherichia coli using the standard method (Brenner, 1974).

\section{Plasmids}

Pacox-1.1::acox-1.1 was constructed by inserting a genomic DNA, which includes a 1.3-kb region of the acox-1.1 promoter, the acox-1.1 coding region, and a $187 \mathrm{bp}$ of the $3^{\prime}$ untranslated region (3'UTR), into pCR2.1.

Pacox-1.1::n/s:::gfp:: 3'UTR (acox-1.1) was constructed by replacing the acox-1.1 coding region of the Pacox-1.1::acox-1.1 plasmid with NLS-GFP region in pPD95.67. The cDNAs used in this study were isolated from the PACT cDNA library (Sakamoto et al., 2005). Punc-25::acox-1.1 (cDNA), Punc-25::acox-1.2 (cDNA), and Punc-25::daf-22 (cDNA) plasmids were constructed by inserting each cDNA into the pSC325 vector. pPD95.75-Pges-1 (Inoue et al., 2005), pPD52.102, and pPD95.75 vectors were used to construct Pges-1::acox-1.1, Pmec-7::acox-1.1, Pmec-7::acox-1.2, and Pmec-7::gfp, respectively. Psrg-36::srg-36::s/2::gfp and Psrg-37::srg-37::s/2::gfp plasmids (McGrath et al., 2011) are a generous gift from Dr. Cori Bargmann. Punc-25::srg-36::s/2::gfp and Punc-25::srg-37::s/2::gfp were constructed by replacing the srg-36 promoter region of Psrg-36::srg-36::s/2::gfp and the srg-37 promoter region of Psrg-37::srg-37::s/2::gfp with the unc-25 promoter of pSC325. Punc-25::nes::tdTomato was constructed by replacing the CFP coding region of 
Shimizu et al., 9

Punc-25::nes::cfp (Hisamoto et al., 2018) with tdTomato cDNA (Clontech).

Pmyo-2::dsred-monomer have been previously described (Li et al., 2012).

Promoter regions for the analysis of gene expression patterns were determined by confirming their ability to rescue the phenotype of the corresponding mutant when combined with protein-coding sequences.

\section{Transgenic animals}

Transgenic animals were obtained using the standard $C$. elegans microinjection method (Table 1; Mello et al., 1991). Pmyo-2::dsred-monomer,

Pacox-1.1::acox-1.1, Pges-1::acox-1.1, Punc-25::acox-1.1, Pmec-7::acox-1.1, Punc-25::acox-1.2, Pmec-7::acox-1.2, Pmec-7::gfp, Pacox-1.1::nls::gfp, Punc-25:::daf-22, Psrg-36:::srg-36::sl2::gfp, Psrg-37::srg-37::sl2:::gfp, Punc-25::srg-36::s/2:::gfp, Punc-25::srg-37::s/2:::gfp, and Punc-25::nes::tdTomato plasmids were used in kmEx1551 [Pacox-1.1::acox-1.1 (25 ng/ $\mu \mathrm{l})+$

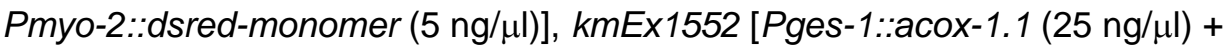

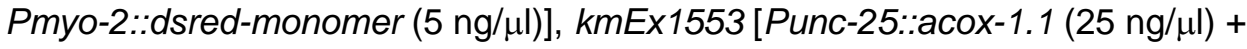

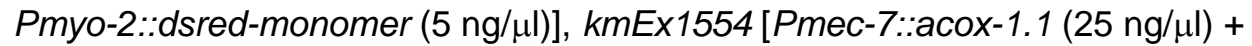

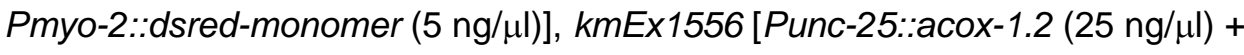

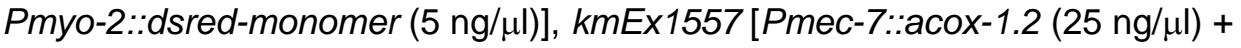

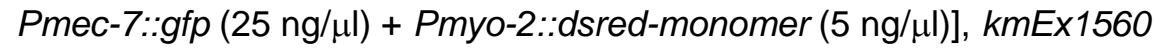
[Pacox-1.1::n/s::gfp $(25 \mathrm{ng} / \mathrm{\mu l})+$ Punc-25::nes::tdTomato $(25 \mathrm{ng} / \mathrm{\mu l})+$

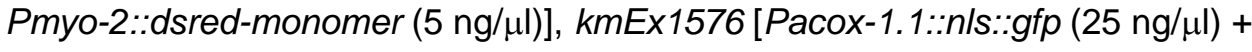

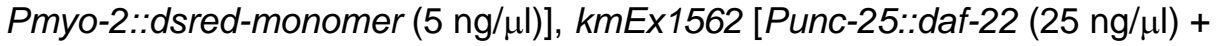
Pmyo-2::dsred-monomer (5 ng/ul)], kmEx1564 [Psrg-36::srg-36::s/2::gfp (25 $\mathrm{ng} / \mathrm{\mu l})+$ Pmyo-2::dsred-monomer (5 ng/ul)], kmEx1565 [Psrg-37::srg-37::s/2:::gfp

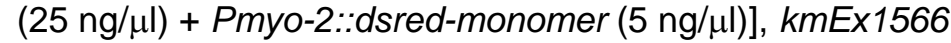

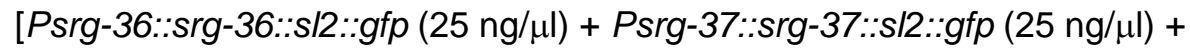




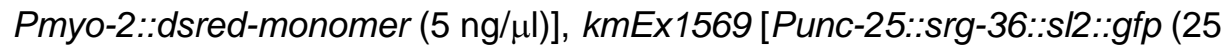

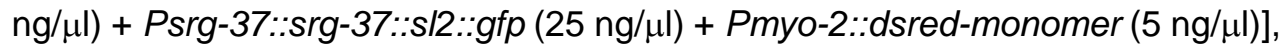

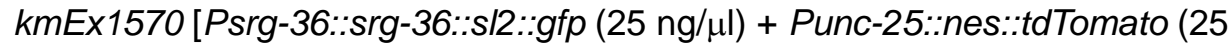
$\mathrm{ng} / \mu \mathrm{l})+$ Pmyo-2::dsred-monomer (5 ng/ $\mu \mathrm{l})]$, kmEx1573 [Punc-25::srg-36::sl2::gfp (25 ng/ $\mu \mathrm{l})+$ Pmyo-2::dsred-monomer $(5 \mathrm{ng} / \mu \mathrm{l})]$, and

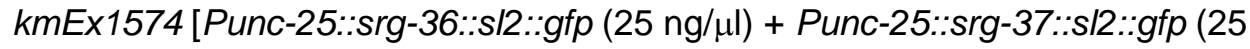
$\mathrm{ng} / \mu \mathrm{l})+$ Pmyo-2::dsred-monomer $(5 \mathrm{ng} / \mu \mathrm{l})]$, respectively.

\section{Generation of the acox-1.4(km92) mutation using CRISPR-Cas9}

The acox-1.4(km92) mutation was generated using the previously described CRISPR-Cas9 system (Dokshin et al., 2018). The CRISPR guide RNA [5'-CCCGUUCCUCGGUGAGAUCCGUUUUAGAGCUAUGCU-3'] was synthesized (Integrated DNA Technologies: IDT) and co-injected with the trans-activating CRISPR RNA (IDT), Streptococcus pyogenes Cas9 3NLS (IDT) protein, and the pRF4(rol-6d) plasmid into the KU501 strain. Subsequently, each F1 organism carrying the transgene was transferred onto a new dish and used for single-worm PCR, followed by DNA sequencing to detect the mutations. The acox-1.4(km92) mutation is a 5-bp deletion in exon 1 of the acox-1.4 gene, causing a frameshift and premature stop codon in exon 1.

\section{Microscopy}

Standard fluorescent images of transgenic animals were observed under a 100x objective using a Nikon ECLIPSE E800 fluorescent microscope and photographed using a Zyla CCD camera. Confocal fluorescent images were taken using a Zeiss LSM-800 confocal laser scanning microscope. For analyzing the expression of acox-1.1 or srg-36 in GABAergic neurons, more than ten axons were analyzed and gene expression was examined every $30 \mathrm{~min}$ for $5 \mathrm{~h}$ 
220

after injury.

\section{Axotomy}

Axotomy and microscopy were performed as previously described (Li et al., 2012). Animals were subjected to axotomy at the $L 4$ or young adult stage. The imaged commissures that had growth cones or small branches present on the proximal fragment were counted as "regenerated." Proximal fragments that showed no change after $24 \mathrm{~h}$ were counted as "no regeneration." A minimum of 15 individuals with 1-3 axotomized commissures were observed for most experiments. Most of the animals with the same genotype regenerated a similar number of axons.

\section{Pheromone treatment}

Ascr\#5, synthesized as described previously (Butcher et al., 2008), was dissolved in DMSO and diluted in M9 media to a final concentration of $1 \mu \mathrm{M}$. After incubating young adult stage animals in this solution for $6 \mathrm{~h}$, axons were cut with a laser and incubated on NGM plates containing ascr\#5 $(1 \mu \mathrm{M})$ for $24 \mathrm{~h}$ before microscopic observation. For the dauer assay, embryos were incubated on NGM plates containing ascr\#5 $(1 \mu \mathrm{M})$ and the OP50 strain for 3 days at $25^{\circ} \mathrm{C}$, and then the numbers of dauer and non-dauer larvae were counted. The rate of dauer formation was calculated by dividing the number of dauer larvae by the total number of dauer and non-dauer larvae.

\section{Phylogenetic analysis}

Evolutionary relationships among candidate genes were determined by constructing neighbor-joining phylogenetic trees using the MEGAX software (Stecher et al., 2020). Evolutionary distances were calculated using the Poisson 
correction method (Zuckerkandl and Pauling, 1965).

\section{Experimental design and statistical analyses}

250 None of the experiments were randomized, and researchers were not blinded to the group assignments during the experiments and evaluation of results. The sample size was determined based on previous studies that assayed axon regeneration in the GABAergic neurons of $C$. elegans (Yanik et al., 2004; Hammarlund et al, 2009). Approximately 50 axons per animal per group were scored. With this sample size, a 30\% difference in axon regeneration was detected with an $80 \%$ probability of detection calculated by the Fisher's exact test based on the sample size. However, due to issues (small body size, thin axons, and weak GFP expression for unknown reasons) originating from specific mutants, it was sometimes challenging to excise the same number of axons in all groups. Statistical analysis was conducted as described in a previous study (Pastuhov et al., 2012). Confidence intervals (95\%) were calculated using the modified Wald test, and two-tailed $P$-values were calculated using Fisher's exact test on GraphPad QuickCalcs were considered statistically significant. 
Shimizu et al., 13

266

267

268

269

270

271

272

273

274

275

276

\section{Results}

\section{Identification of SVH-18/SRX-16}

In C. elegans, axon regeneration is regulated by the EGL-30 Gq $\alpha-J N K$ pathway (Fig. 1A; Sakai et al., 2021). However, the GPCRs that act upstream of EGL-30 remain unknown. To identify additional components functioning in the JNK pathway, we previously conducted a genome-wide RNAi screening for suppressors of $v h p-1$ lethality and isolated 92 svh genes (Li et al., 2012; Shimizu et al., 2021). Indeed, we isolated the egl-30 gene as svh-12 (Shimizu et al., 2021). To identify GPCRs involved in the EGL-30 signaling pathway, we examined whether svh genes encode GPCRs. We identified the svh-18 gene, which encodes SRX-16, a predicted GPCR chemoreceptor in the srx gene family (Fig. $1 B, C$ ). To determine the effect of srx-16 on axon regeneration, we assayed regrowth after laser axotomy in $\mathrm{y}$-aminobutyric acid (GABA)-releasing D-type motor neurons, which extend their axons from the ventral to the dorsal nerve cord (Yanik et al., 2004; Hammarlund et al., 2009). In young adult wild-type animals, about $62 \%$ of laser-severed axons could initiate regeneration within $24 \mathrm{~h}$ (Fig. 1D; Table 2). We found that the srx-16(tm7585) deletion mutation (Fig. 1B) did not affect axon regeneration (Fig. 1D; Table 2). Therefore, the function of SRX-16 in the JNK pathway is to regulate larval growth but not axon regeneration.

\section{Enzymes involved in ascaroside pheromone biosynthesis participate in} axon regeneration

There are 96 members of the SRX family (Robertson and Thomas, 2006). Of which, only SRX-43 and SRX-44 act as GPCRs for the indolated ascaroside, icas\#9 (IC-asc-C5; C5) (Greene et al., 2016a, 2016b), whereas the functions of 
the other members are still unknown. The generated phylogenetic tree shows that SRX-16 is similar to SRX-43/SRX-44 (Fig. 1C), raising the possibility that the ascaroside pheromone may function as a signal for activating the EGL-30 pathway, which promotes axon regeneration. To test this possibility, we examined whether mutants lacking the enzymes involved in ascaroside production would affect axon regeneration. Ascarosides in $C$. elegans are synthesized by the FA $\beta$-oxidation pathway consisting of ACOXs, MAOC-1, DHS-28, and DAF-22 (Fig. 2A). We investigated the effects of deletion mutations in acox-1.1 and daf-22 (Fig. 2B) on the regeneration of D-type motor neurons. The acox-1.1 gene encodes one of the ACOX enzymes, and the acox-1.1(ok2257) deletion has been verified to abolish the ACOX-1.1 function (Fig. 2A,B; Joo et al., 2010; Zhang et al., 2016, 2018). The daf-22 gene encodes an ortholog of the human sterol carrier protein, SCPx, and the daf-22(ok693) deletion results in the loss of its enzymatic function (Fig. 2A,B; Butcher et al., 2009; Joo et al., 2009). The acox-1.1(ok2257) deletion or the daf-22(ok693) deficiency causes the accumulation of large fat granules in the intestine, reduced growth rate, and decreased brood size (Joo et al., 2009, 2010). On comparison with wild-type animals, we found that the frequency of axon regeneration after laser axotomy was reduced in acox-1.1(ok2257) and daf-22(ok693) mutants (Fig. 2C; Table 2). To verify that the acox-1.1 mutation causes this defect in axon regeneration, we generated the transgene Pacox-1.1::acox-1.1, which contains the entire genomic acox-1.1 coding region, its promoter, and the $3^{\prime} \mathrm{UTR}$. Introduction of Pacox-1.1::acox-1.1 into acox-1.1(ok2257) mutants significantly rescued the regeneration defect (Fig. 2C; Table 2).

To test whether ACOX-1.1 functions in the EGL-30 signaling pathway, we examined the genetic interactions between acox-1.1 and egl-30. EGL-30 is the 
C. elegans $\mathrm{Gq \alpha}$, and we employed two egl-30 alleles, egl-30(ad805) and egl-30(tg26) (Fig. 2B). The egl-30(ad805) loss-of-function (If) mutation is a mutation in the splice acceptor site that reduces the number of copies of full-length EGL-30 (Brundage et al., 1996). In contrast, the egl-30(tg26) gain-of-function (gf) mutation is a missense mutation that constitutively activates EGL-30 function by replacing the conserved Arg-243 with glutamine (Doi and Iwasaki, 2002). We found that the defect in axon regeneration caused by the acox-1.1(ok2257) mutation was not enhanced by introducing the egl-30(If) mutation (Fig. 2C; Table 2). This result suggests that ACOX-1.1 and EGL-30 act in the same pathway. Moreover, the activating egl-30(gf) mutation could suppress the acox-1.1 phenotype (Fig. 2C; Table 2), suggesting that EGL-30 functions downstream of ACOX-1.1. These results support the possibility that ACOX-1.1 regulates axon regeneration through the EGL-30 pathway.

\section{Expression pattern of acox-1.1}

To investigate the location of ACOX-1.1 when it regulates axon regeneration, we examined the expression pattern of acox-1.1. We constructed a transgene, Pacox-1.1::nls:::gfp::3'UTR (acox-1.1), which consists of the acox-1.1 promoter, nuclear localization signal (NLS), fluorescent protein GFP, and acox-1.1 3'UTR. The acox-1.1 gene functions in the intestine and hypodermis, where it contributes to the biosynthesis of ascaroside pheromones (Joo et al., 2010). Consistent with this, the acox-1.1 gene is exclusively expressed in intestinal cells but not in D-type neurons. GFP expression was still not observed in D neurons after axon injury (Fig. 3A).

Although acox-1.1 is expressed in the intestine, we found that the expression of acox-1.1 cDNA from the ges-1 promoter in the intestine could not rescue the axon regeneration defect in acox-1.1(ok2257) mutants (Fig. 3B; Table 2). Recent 
observations suggest that peroxisomal FA $\beta$-oxidation may have an as-yet-unexplored function in neurons (Park and Paik, 2017). Indeed, we found that the acox-1.1 deficiency was rescued by expression of acox-1.1 cDNA from the unc-25 promoter in D-type motor neurons but not from the mec-7 promoter in touch neurons (Fig. 3B; Table 2). Similar to acox-1.1, the expression of daf-22 with the unc-25 promoter suppressed the daf-22 defect (Fig. 3B; Table 2). These results suggest that ACOX-1.1 and DAF-22 promote the regeneration of damaged neurons in a cell-autonomous manner.

\section{Ascaroside ascr\#5 promotes axon regeneration}

We next evaluated which ascaroside regulates axon regeneration. In C. elegans, ascarosides are grouped into two main classes: $\omega$-ascarosides and ( $\omega$-1)-ascarosides (Fig. 2A; Butcher, 2017). They are biosynthesized via two parallel $\beta$-oxidation pathways, each involving different ACOX enzymes (Fig. 4A; Zhang et al., 2015, 2016, 2018). The former pathway involves ACOX-1.2, and the latter depends on ACOX-1.3, ACOX-1.4, and ACOX-3. ACOX-1.1 and DAF-22 are required in both pathways. The acox-1.2(gk386052) and acox-3(gk203391) mutations contain nonsense mutations, and they are probably null mutations because they result in premature stop codons at Trp-496 and Trp-395, respectively (Fig. 4B). We found that the acox-1.2(gk386052) mutation reduced axon regeneration (Fig. 4C; Table 2). In contrast, the acox-1.3(tm5192) deletion, which disrupts the ACOX-1.3 function (Fig. 4B; Zhang et al., 2015, 2016) or the acox-3(gk203391) mutation had little effect on axon generation (Fig. 4C; Table 2). Furthermore, a putative null mutation, acox-1.4(km92) (Fig. 4B), did not affect axon regeneration (Fig. 4C; Table 2). These results suggest that $\omega$-ascarosides are important for axon regeneration. 
Since ACOX-1.2 influences the production of an ascaroside with a short $\omega$-side chain, i.e., the dauer pheromone asc- $\omega \mathrm{C} 3$ (C3; ascr\#5) (Fig. 2A; Zhang et al., 2015), we examined the effect of synthetic ascr\#5 on axon regeneration. First, we supplied ascr\#5 externally to adult-stage acox-1.2 mutants and then determined the axon regeneration frequency. We found that the presence of ascr\#5 was sufficient to induce dauer formation in acox-1.2(gk386052) mutant larvae when added from an embryo (Fig. $5 A, B$ ), and it significantly rescued the axon regeneration defect in acox-1.2(gk386052) mutants when introduced the young adult stage (Fig. $5 C, D$; Table 2). These results indicate that ascr\#5 is involved in axon regeneration.

\section{acox-1.2 expression inside the injured neuron is required for axon}

\section{regeneration}

Expression of acox-1.2 with the unc-25 promoter in D neurons suppressed the acox-1.2 defect (Fig. 4C; Table 2), suggesting that ACOX-1.2 regulates axon regeneration in a cell-autonomous manner. Therefore, we examined whether acox-1.2 expression inside the injured neuron is required for axon regeneration. To test this possibility, we expressed the acox-1.2 gene in touch neurons using the mec-7 promoter. Touch neuron axons run parallel to the body axis and intersect perpendicularly to D-type neuron axons (Fig. 6A). Expression of acox-1.2 in touch neurons could not rescue the acox-1.2 deficiency in D-type motor neuron regeneration (Fig. 6B; Table 2). This result is consistent with the possibility that ACOX-1.2 functions cell-autonomously in axon regeneration. On the other hand, when both touch and D neurons were severed simultaneously in acox-1.2(gk386052) mutants expressing acox-1.2 in touch neurons, the regeneration defect of $D$ neurons was suppressed (Fig. 6B; Table 2). In wild-type animals, simultaneous damage to the axons of touch and D neurons did not 
affect the frequency of $D$ neuron regeneration (Fig. 6B; Table 2). Altogether, these results suggest that ACOX-1.2 induces ascr\#5 production in axonally injured touch neurons and the produced and secreted ascr\#5 acts on damaged D neurons to promote regeneration.

\section{The SRG-36/SRG-37 GPCRs of ascr\#5 are involved in axon regeneration} srg-36 and srg-37genes are two members of the nematode-specific GPCR family that encode receptors for ascr\#5 (McGrath et al., 2011). We therefore determined whether these GPCRs are involved in axon regeneration. Because the srg-36 and srg-37 genes are adjacent to each other in the genome, the kyIR95 allele deletes both the genes (Fig. 7A). We found that at the young adult stage, the frequency of axon regeneration was reduced in srg-36 srg-37(kylR95) (Fig. 7B; Table 2). SRG-36 and SRG-37 function redundantly to support dauer formation in response to ascr\#5 (McGrath et al., 2011). To investigate whether SRG-36 and SRG-37 also show redundancy in regulating axon regeneration or whether both are required for ascr\#5 signaling, the reporter transgenes Psrg-36::srg-36::s/2::gfp and Psrg-37::srg-37::s/2::gfp (Fig. 7A) were introduced into srg-36 srg-37(kyIR95) mutants and we measured the axon regeneration frequency. These transgenes contain bicistronic fusion genes and are functional (McGrath et al., 2011). Transgenic animals expressing either of the two transgenes were defective in axon regeneration, but introducing both transgenes together rescued the srg-36 srg-37 (kyIR95) phenotype (Fig. 7C; Table 2). These results indicate that both SRG-36 and SRG-37 are required for axon regeneration after laser axotomy.

To confirm that SRG-36/SRG-37 act as receptors for ascr\#5 to promote axon regeneration, we examined the effect of ascr\#5 addition on axon regeneration in srg-36 srg-37 (kyIR95) mutants. As observed previously (McGrath et al., 2011), 
srg-36 srg-37 (kyIR95) mutants were resistant to dauer formation induced by ascr\#5 (Fig. 5A,B). This result is in parallel with the fact that SRG-36/SRG-37 are GPCRs of ascr\#5. Similarly, we found that the presence of ascr\#5 could not rescue the axon regeneration defect in srg-36 srg-37 (ky/R95) mutants (Fig. $5 C, D$; Table 2). These results indicate that ascr\#5 promotes axon regeneration via SRG-36/SRG-37.

To determine whether SRG-36 functions in D-type motor neurons, we examined the expression pattern of srg-36 using the Psrg-36::srg-36::s/2::gfp reporter gene (McGrath et al., 2011). Previous studies have shown that srg-36 is strongly expressed in ASI neurons but are weakly or inconsistently expressed in several other neurons (McGrath et al., 2011). At the young adult stage, animals carrying Psrg-36::srg-36::sl2::gfp did not show GFP expression in D-type motor neurons. In addition, no GFP expression was observed in D neurons after axon injury (Fig. 8). Therefore, to confirm that SRG-36 acts in D-type motor neurons, we expressed srg-36::s/2::gfp from the unc-25 promoter (Fig. 7A) in srg-36 srg-37 (kyIR95) mutants carrying Psrg-37::srg-37::s/2::gfp. We found that the srg-36 defect in axon regeneration was rescued by the expression of srg-36 by the unc-25 promoter in D-type motor neurons (Fig. 7D; Table 2). However, the expression of Punc-25::srg-36::s/2::gfp alone could not rescue the srg-36 srg-37 (kyIR95) mutant phenotype (Fig. 7D; Table 2). This is consistent with the idea that both SRG-36 and SRG-37 are required for axon regeneration. Furthermore, the expression of $s r g-37:: s / 2:: g$ fp from the unc-25 promoter (Fig. 7A) rescued the srg-36 srg-37 (kyIR95) mutant phenotype with the Punc-25::srg-36::s/2::gfp construct (Fig. 7D; Table 2). These results demonstrate that SRG-36 and SRG-37 regulate axon regeneration in injured D-type motor neurons after laser axotomy in a cell-autonomous manner. 


\section{SRG-36 and SRG-37 function in the EGL-30 pathway to promote axon} regeneration

Finally, we investigated whether SRG-36/SRG-37 GPCRs function in the EGL-30-mediated pathway to promote axon regeneration. We have previously demonstrated that the CED-10 Rac type GTPase-MAX-2 and EGL-30 Gq TPA-1 PKC pathways regulate axon regeneration mainly at the $L 4$ and young adult stages, respectively (Pastuhov et al., 2012). Furthermore, it has been shown that max-2 is expressed during early development, but not at the young adult stage (Lucanic et al., 2006). This suggests that TPA-1 replaces MAX-2 to activate MLK-1 during axon regeneration at the adult stage (Fig. $1 A$ ). Therefore, we examined the relationship between life stage and axon regeneration in srg-36 srg-37(kyIR95) mutants. We found that axon regeneration in srg-36 srg-37(kyIR95) mutants was reduced only at young adult animals and not at L4 larvae, a phenotype similar to that observed in egl-30(If) mutants (Fig. 7B,E; Table 2). Thus, SRG-36 and SRG-37 participate in axon regeneration specifically at the adult stage.

We also examined the genetic interactions of srg-36 srg-37 with egl-30. We found that the regeneration defect in srg-36 srg-37(kyIR95); egl-30(If) triple mutants was not greater than the regeneration defect in srg-36 srg-37(kyIR95) or egl-30(If) mutants (Fig. 7B; Table 2). This result supports the possibility that SRG-36/SRG-37 function in the EGL-30 signaling pathway. Furthermore, the srg-36 srg-37 phenotype was suppressed by the egl-30(gf) mutation (Fig. 7B; Table 2), suggesting that SRG-36/SRG-37 function upstream of EGL-30. Thus, SRG-36/SRG-37 GPCRs promote axon regeneration by activating the EGL-30 Gqa pathway. 
Shimizu et al., 21

\section{Discussion}

Pheromones are molecules secreted by individuals that can induce changes in the behavior and development of different animals of the same species. $C$. elegans secretes ascarosides, which constitute a conserved family of signaling molecules, as pheromones to communicate with other animals and to coordinate population development and behavior (Peso et al., 2015). Originally, ascarosides were identified as components of the dauer pheromone, which is the population density signal. High population density result in ascaroside accumulation, which in combination with additional environmental stimuli, such as limited food availability and temperature stress, promotes larval arrest in the dauer stage (Golden and Riddle, 1984). In this study, we found that ascaroside signaling regulates neural processes in $C$. elegans. In particular, we show that the loss of ascaroside production impairs axon regeneration. Furthermore, GPCR sensing of ascaroside regulates axon regeneration via the EGL-30 Gq $\alpha$ signaling pathway (Fig. 9).

The primary site of ascaroside biosynthesis appears to be the intestine, and ascaroside is also likely to be excreted via the intestine (Butcher et al., 2009). However, the expression of the acox-1.1 gene in the intestine fails to rescue the axon regeneration defect in acox-1.1 mutants, whereas its expression in injured neurons can restore the acox-1.1 deficiency. Thus, ACOX-1.1 promotes the regeneration of damaged neurons in a cell-autonomous manner. Furthermore, we demonstrated that acox-1.2 expression in injured neurons has an important function in regulating axon regeneration. Expression of acox-1.2 in touch neurons does not rescue the acox-1.2 deficiency in D-type motor neuron regeneration. However, in acox-1.2 mutants expressing acox-1.2 in touch neurons, simultaneous laser ablation of axons of $D$ and touch neurons rescues 
the regeneration defect of $D$ neurons. These results suggest that ACOX-1.2 expressed in the damaged touch neuron induces the production of ascaroside, which in turn acts on the nearby damaged $D$ neuron to induce regeneration. Thus, ascaroside is synthesized in axotomized neurons and is required for axon regeneration, suggesting that ascaroside regulates axon regeneration as a non-pheromone signal. Since axotomized neurons gain the ability to synthesize ascaroside in response to axon injury, it appears that transcriptional regulation is necessary to ensure that sufficient amounts of ascaroside are available when axons are damaged. Therefore, it is important to identify the transcription factors that regulate the transcription of genes for ascaroside synthesis after axon injury. Since our svh screening revealed genes that encode transcription factors (Shimizu et al., 2021), analysis of these svh genes will shed new light into the mechanism underlying the transcription of ascaroside synthesis genes regulated in response to axon injury.

What is the ascaroside that regulates axon regeneration? We show that mutations in acox-1.1, daf-22, and acox-1.2 are defective in axon regeneration, whereas acox-3, acox-1.3, or acox-1.4 mutations have little effect on axon regeneration. ACOX-1.1, DAF-22, and ACOX-1.2 participate in the $\beta$-oxidation cycles that shorten $\omega$-ascarosides, with ACOX-1.2 specifically participating in the last of these $\beta$-oxidation cycles, which generates ascr\#5 (Zhang et al., 2018). An acox-1.2 mutant only shows defects in the production of ascr\#5 and not in that of other ascarosides. Thus, ascr\#5 is a candidate ascaroside responsible for promoting axon regeneration after axon injury. Indeed, ascr\#5 recovers the ability of the acox-1.2 mutant to regenerate axons. The ascaroside signal is sensed by GPCRs in specific chemosensory neurons (Butcher, 2017). srg-36 and srg-37 genes encode GPCRs for ascr\#5 (McGrath et al., 2011). We found that the srg-36 srg-37 double mutation decreases axon regeneration ability. This 
indicates that $C$. elegans utilizes SRG-36/SRG-37 GPCRs to recognize ascr\#5 for initiating axon regeneration after axon injury. The expression of GPCRs for sensing ascarosides is clearly predominant in sensory neurons, whereas srg-36/srg-37 function in injured motor neurons. In most cases, each GPCR is strictly expressed in different cell types, contributing to their distinct and cell type-specific responses to internal signals (Rohrer and Kobilka, 1998). Furthermore, recent studies have revealed that the expression of several chemoreceptor genes, such as srh-234 and odr-10, is regulated by environmental stimuli (Gruner et al., 2014; Ryan et al., 2014). These results raise the possibility that SRG-36 and SRG-37 are produced in response to nerve injury, activating ascar\#5 signaling via the autocrine system and inducing axon regeneration.

The ascr\#5-specific SRG-36 and SRG-37 provide an example for highly structure-specific ascaroside receptors (McGrath et al., 2011). Our finding that SRG-36 and SRG-37 do not function redundantly in axon regeneration suggests that heterodimerization of SRG-36 and SRG-37 may be necessary to form a functional complex for signal transduction. Recent studies have suggested that GPCRs associate as dimers or higher-order oligomers (Lause, 2010). For example, SRBC-64/SRBC-66 function as part of receptor GPCR dimers or higher-order oligomers, including more specific receptors, such as DAF-37 (Park et al., 2012). Thus, the complex ascaroside signaling properties may partly be due the interaction of several different ascaroside receptors that bind directly to ascarosides.

Although ascarosides are specific to nematodes, other similar lipid molecules may contribute to promoting axon regeneration in mammals. Peroxisome proliferator-activated receptor $\alpha$ (PPAR $\alpha$ ) induces ACOXs gene expression in mammals (Marcus et al., 1993). In rat dorsal root ganglion neurons, 
axonal damage increases PPAR protein levels, causing PPAR transport from the distal axons to the nucleus and promoting neuronal regeneration (Lezana et al., 2016). Furthermore, thiazolidinedione, a PPAR agonist, promotes axonal growth in rat hippocampal neurons by activating the JNK pathway in a PPAR $\alpha$-dependent manner (Quintanilla et al., 2013). Therefore, it is possible that lipid metabolites produced by ACOX enzymes in the peroxisome may induce JNK activation and promote axon regeneration.

Each GPCR couples preferentially with a functionally distinct class of $\mathrm{G \alpha}$ proteins (Möller et al., 2001). In this study, we found that SRG-36/SRG-37 GPCRs activate EGL-30 Gq $\alpha$ and promote axon regeneration. We have recently demonstrated that AEA modulates the axon regeneration response of GABAergic motor neurons after laser axotomy (Pastuhov et al., 2012). AEA functions as an inhibitory signal for axon regeneration, which is transmitted through the NPR-19/NPR-32 GPCRs-GOA-1 Goo pathway and antagonizes EGL-30 Gqa. Therefore, axon regeneration in C. elegans appears to be determined by the balance of stimulatory and inhibitory signals, such as ascaroside and $A E A$, which are transduced by $G \alpha$ protein signal transduction pathways (Fig. 9). Thus, axon regeneration after axonal injury in $C$. elegans is regulated by the $\mathrm{G}_{0} \alpha-\mathrm{G}_{\mathrm{q}} \alpha$ signaling pathway. 
Shimizu et al., 25

\section{References}

Brenner S (1974) The genetics of Caenorhabditis elegans. Genetics 77:71-94.

Brundage L, Avery L, Katz A, Kim UJ, Mendel JE, Sternberg PW, Simon MI (1996) Mutations in a C. elegans Gqa gene disrupt movement, egg laying, and viability. Neuron 16:999-1009.

Butcher RA, Fujita M, Schroeder FC, Clardy J (2007) Small-molecule pheromones that control dauer development in Caenorhabditis elegans. Nat Chem Biol 3:420-422.

Butcher RA, Ragains JR, Kim E, Clardy J (2008) A potent dauer pheromone component in Caenorhabditis elegans that acts synergistically with other components. Proc Natl Acad Sci USA 105:14288-14292.

Butcher RA, Ragains JR, Li W, Ruvkun G, Mak HY (2009) Biosynthesis of the Caenorhabditis elegans dauer pheromone. Proc Natl Acad Sci USA 106:1875-1879.

Butcher RA (2017) Small-molecule pheromones and hormones controlling nematode development. Nat Chem Biol 13:577-586.

Doi M, Iwasaki K (2002) Regulation of retrograde signaling at neuromuscular junctions by the novel C2 domain protein AEX-1. Neuron 33:249-259.

Dokshin GA, Ghanta KS, Piscopo KM, Mello CC (2018) Robust genome editing with short single-stranded and long, partially single-stranded DNA donors in Caenorhabditis elegans. Genetics 210:781-787.

Golden JW, Riddle DL (1984) The Caenorhabditis dauer larva: developmental effects of pheromone, food, and temperature. Dev Biol 102:368-378.

Greene JS, Brown M, Dobosiewicz M, Ishida IG, Macosko EZ, Zhang X, Butcher RA, Cline DJ, McGrath PT, Bargmann Cl (2016a) Balancing selection shapes density-dependent foraging behaviour. Nature 539:254-258. 
Shimizu et al., 26

606

607

608

609

Greene JS, Dobosiewicz M, Butcher RA, McGrath PT, Bargmann Cl (2016b) Regulatory changes in two chemoreceptor genes contribute to a Caenorhabditis elegans QTL for foraging behavior. eLife 5:e21454.

Gruner M, Nelson D, Winbush A, Hintz R, Ryu L, Chung SH, Kim K, Gabel CV, van der Linden AM (2014) Feeding state, insulin and NPR-1 modulate chemoreceptor gene expression via integration of sensory and circuit inputs. PLoS Genet 10:e1004707.

Hammarlund M, Nix P, Hauth L, Jorgensen EM, Bastiani M (2009) Axon regeneration requires a conserved MAP kinase pathway. Science 323:802806.

Hisamoto N, Matsumoto K (2017) Signal transduction cascades in axon regeneration: insights from C. elegans. Curr Opin Genet Dev 44:54-60. Hisamoto N, Tsuge A, Pastuhov SI, Shimizu T, Hanafusa H, Matsumoto K (2018) Phosphatidylserine exposure mediated by $A B C$ transporter activates the integrin signaling pathway promoting axon regeneration. Nat Commun 9:3099.

Inoue H, Hisamoto N, An JH, Oliveira RP, Nishida E, Blackwell TK, Matsumoto K (2005) The C. elegans p38 MAPK pathway regulates nuclear localization of the transcription factor SKN-1 in oxidative stress response. Genes Dev 19:2278-2283.

Joo HJ, Yim YH, Jeong PY, Jin YX, Lee JE, Kim H, Jeong SK, Chitwood DJ, Paik YK (2009) Caenorhabditis elegans utilizes dauer pheromone biosynthesis to dispose of toxic peroxisomal fatty acids for cellular homoeostasis. Biochem J 422:61-71. Joo HJ, Kim KY, Yim YH, Jin YX, Kim H, Kim MY, Paik YK (2010) Contribution of the peroxisomal acox gene to the dynamic balance of daumone production in Caenorhabditis elegans. J Biol Chem 285:29319-29325. 
Kaplan A, Tone SO, Fournier A (2015) Extrinsic and intrinsic regulation of axon regeneration at a crossroads. Front Mol Neurosci 8:27.

Lause MJ (2010) Dimerization in GPCR mobility and signaling. Curr Opin Pharmacol 10:53-58.

Lezana JP, Dagan SY, Robinson A, Goldstein RS, Fainzulber M, Bronfman FC, Bronfman M (2016) Axonal PPARy promotes neuronal regeneration after injury. Dev Neurobiol 76:688-701.

Li C, Hisamoto N, Nix P, Kanao S, Mizuno T, Bastiani M, Matsumoto K (2012) The growth factor $\mathrm{SVH}-1$ regulates axon regeneration in $C$. elegans via the JNK MAPK cascade. Nat Neurosci 15:551-557.

Lucanic M, Kiley M, Ashcroft N, L'etoile N, Cheng HJ (2006) The Caenorhabditis elegans P21-activated kinases are differentially required for UNC-6/netrin-mediated commissural motor axon guidance. Development 133:4549-4559.

Mahar M, Cavalli V (2018) Intrinsic mechanisms of neuronal axon regeneration. Nat Rev Neurosci 19:323-337.

Marcus SL, Miyata KS, Zhang B, Subramani S, Rachubinski RA, Capone JP (1993) Diverse peroxisome proliferator-activated receptors bind to the peroxisome proliferator-responsive elements of the rat hydratase/dehydratase and fatty acid acyl-CoA oxidase genes but differentially induce expression. Proc Natl Acad Sci USA 90:5723-5727.

McGrath PT, Xu Y, Ailion M, Garrison JL, Butcher RA, Bargmann CI (2011) Parallel evolution of domesticated Caenorhabditis species targets pheromone receptor genes. Nature 477:321-325.

Mello CC, Kramer JM, Stinchcomb D, Ambros V (1991) Efficient gene transfer in C. elegans: extrachromosomal maintenance and integration of transforming sequences. EMBO J 10:3959-3970. 
Shimizu et al., 28

Mizuno T, Hisamoto N, Terada T, Kondo T, Adachi M, Nishida E, Kim DH, Ausubel FM, Matsumoto K (2004) The Caenorhabditis elegans MAPK phosphatase VHP-1 mediates a novel JNK-like signaling pathway in stress response. EMBO J 23:2226-2234.

Möller S, Vilo J, Croning MDR (2001) Prediction of the coupling specificity of G protein coupled receptors to their $\mathrm{G}$ proteins. Bioinformatics 17:S174-S181.

Park D, O'Doherty I, Somvanshi RK, Bethke A, Schroeder FC, Kumar U, Riddle DL (2012) Interaction of structure-specific and promiscuous G-protein coupled receptors mediates small-molecule signaling in Caenorhabditis elegans. Proc Natl Acad Sci USA 109:9917-9922.

Park JY, Joo HJ, Park S, Paik YK (2019) Ascaroside pheromones: chemical biology and pleiotropic neuronal functions. Int J Mol Sci 20:3898.

Park S, Paik YK (2017) Genetic deficiency in neuronal peroxisomal fatty acid $\beta$-oxidation causes the interruption of dauer development in Caenorhabditis elegans. Sci Rep 7:9358.

Pastuhov SI, Fujiki K, Nix P, Kanao S, Bastiani M, Matsumoto K, Hisamoto N (2012) Endocannabinoid-Go $\alpha$ signalling inhibits axon regeneration in Caenorhabditis elegans by antagonizing Gq $\alpha-P K C-J N K$ signalling. Nat Commun 3:1136.

Peso M, Elgar MA, Barron AB (2015) Pheromonal control: reconciling physiological mechanism and signaling theory. Biol Rev 90:542-559.

Quintanilla RA, Godoy JA, Alfaro I, Cabezas D, von Bernhardi R, Bronfman M, Inestrosa NC (2013) Thiazolidinediones promote axonal growth through the activation of the JNK pathway. PLoS One 8:e65140.

Robertson HM, Thomas JH (2006) The putative chemoreceptor families of $C$. elegans. Wormbook Jan 6:1-12.

Rohrer DK, Kobilka BK (1998) G protein-coupled receptors: functional and 
Shimizu et al., 29

687

688

689

690

691

692

693

694

695

696

697

698

699

700

mechanistic insights through altered gene expression. Phys Rev 78:1-35.

Ryan DA, Miller RM, Lee K, Neal SJ, Fagan KA, Sengupta P, Portman DS (2014) Sex, age, and hunger regulate behavioral prioritization through dynamic modulation of chemoreceptor expression. Curr Biol 24:2509-2517.

Sakai Y, Hanafusa H, Shimizu T, Pastuhov SI, Hisamoto N, Matsumoto K (2021) BRCA1-BARD1 regulates axon regeneration in concert with the Gq $\alpha-D A G$ signaling network. J Neurosci 41:2842-2853.

Sakamoto R, Byrd DT, Brown HM, Hisamoto N, Matsumoto K, Jin Y (2005) The Caenorhabditis elegans UNC-14 RUN domain protein binds to the kinesin-1 and UNC-16 complex and regulates synaptic vesicle localization. Mol Biol Cell 16:483-496.

Shimizu T, Pastuhov SI, Hanafusa H, Sakai Y, Todoroki Y, Hisamoto N, Matsumoto K (2021) Caenorhabditis elegans F-box protein promotes axon regeneration by inducing degradation of the Mad transcription factor. $\mathrm{J}$ Neurosci 41:2373-2381.

Stecher G, Tamura K, Kumar S (2020) Molecular evolutionary genetics analysis (MEGA) for MacOS. Mol Biol Evol 37:1237-1239.

Tedeshi A, Bradke F (2017) Spatial and temporal arrangement of neuronal intrinsic and extrinsic mechanisms controlling axon regeneration. Curr Opin Neurobiol 42:118-127.

von Reuss SH, Dolke F, Dong C (2017) Ascaroside profiling of Caenorhabditis elegans using gas chromatography-electron ionization mass spectrometry. Anal Chem 89:10570-10577.

Yanik MF, Cinar H, Cinar HN, Chisholm AD, Jin Y, Ben-Yakar A (2004) Functional regeneration after laser axotomy. Nature 432:822.

Zhang X, Feng L, Chinta S, Singh P, Wang Y, Nunnery JK, Butcher RA (2015) Acyl-CoA oxidase complexes control the chemical message produced by 
Shimizu et al., 30

714 Caenorhabditis elegans. Proc. Natl. Acad. Sci. USA 112, 3955-3960.

715 Zhang X, Li K, Jones RA, Steven DB, Butcher RA (2016) Structural

716 characterization of acyl-CoA oxidases reveals a direct link between

717 pheromone biosynthesis and metabolic state in Caenorhabditis elegans. Proc

$718 \quad$ Natl Acad Sci USA 113:10055-10060.

719 Zhang X, Wang Y, Perez DH, Jones Lipinski RA, Butcher RA (2018) Acyl-CoA

720 oxidases fine-tune the production of ascaroside pheromones with specific

721 side chain lengths. ACS Chem Biol 13:1048-1056.

722 Zuckerkandl E, Pauling L (1965) Evolutionary divergence and convergence in proteins. Edited in Evolving Genes and Proteins by Bryson V, Vogel HJ 97166. Academic Press, New York. 


\section{Figure Legends}

Figure 1. Characterization of SVH-18/SRX-16.

$\boldsymbol{A}$, The EGL-30 pathway regulating axon regeneration. EGL-30 Gq $\alpha$ activates EGL-8 PLC $\beta$, which in turn generates DAG from phosphatidylinositol bisphosphate $[\mathrm{PI}(4,5) \mathrm{P} 2]$. DAG activates TPA-1 PKC, resulting in activation of the JNK pathway to promote axon regeneration mainly at the young adult stage. The MAPK phosphatase VHP-1 inactivates KGB-1 JNK. $\boldsymbol{B}$, Structure of SRX-16. The black box indicates the transmembrane regions. The bold line underneath indicates the extent of the deleted region in the $\operatorname{tm} 7585$ mutant.

$C$, Phylogenetic tree depicting the genetic relationships among the GPCRs of $C$. elegans. The phylogenetic tree was constructed using the MEGAX software for Mac. SRX-16 was compared with chemoreceptors of the SRG superfamily and other GPCRs whose ligands have been identified. The scale bar represents the evolutionary distance calculated using the Poisson correction method based on the number of amino acid substitutions per site. The similarity (\%) of amino acids between SRX-16 and other GPCRs is shown.

$D$, Percentages of axons that initiated regeneration $24 \mathrm{~h}$ after laser surgery at the young adult stage. The number of axons examined is shown. Error bars indicate $95 \%$ confidence intervals. NS, not significant.

Figure 2. ACOX-1.1 and DAF-22 are required for axon regeneration.

$\boldsymbol{A}$, Ascaroside biosynthesis pathway. Ascarosides fall into two classes- $(\omega-1)$-ascarosides in which the side chain is attached to the ascarylose sugar at the penultimate $(\omega-1)$ position and $\omega$-ascarosides in which the side chain is attached at the terminal $(\omega)$ position. These two ascaroside classes are 
shortened by two $\beta$-oxidation pathways, one involving ACOX-1.1, ACOX-3, ACOX-1.4, and ACOX-1.3 to produce ( $\omega$-1)-ascaroside pheromones (such as those shown in yellow boxes) and another involving ACOX-1.1 and ACOX-1.2 to produce $\omega$-ascaroside pheromones (such as the one shown in the yellow box).

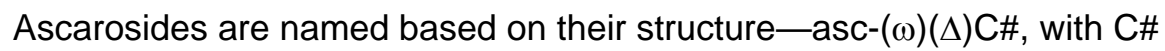
indicating the number of carbons in the side chain, $\omega$ indicating $\omega$-side chain, and $\Delta$ indicating $\alpha-\beta$ unsaturation.

$\boldsymbol{B}$, Structures of ACOX-1.1, DAF-22, and EGL-30. Schematic domain diagrams of $C$. elegans ACOX-1.1, DAF-22, and EGL-30 and their human counterparts are shown. The regions deleted in ok2257 and ok693 are indicated by black bars. The egl-30 loss-of-function (ad805) and gain-of-function ( $\operatorname{tg} 26)$ mutations are shown.

C, Percentages of axons that initiated regeneration $24 \mathrm{~h}$ after laser surgery at the young adult stage. The number of axons examined is shown. Error bars indicate $95 \%$ confidence intervals. ${ }^{*} P<0.05,{ }^{* *} P<0.01$, as determined by Fisher's exact test. NS, not significant.

\section{Figure 3. ACOX-1.1 and DAF-22 regulate axon regeneration in a} cell-autonomous manner.

$\boldsymbol{A}$, Expression pattern of the acox-1.1 gene. Fluorescent and DIC images of animals carrying Pacox-1.1::n/s::gfp::acox-1.1 3'UTR and Punc-25::nes::tdTomato $1 \mathrm{~h}$ after excision are shown. D-type motor neurons are visualized using tdTomato fused to a nuclear export signal (NES) under control of the unc-25 promoter. Blue, yellow, and white arrowheads indicate a severed axon, the corresponding cell body of the injured neuron, and cell nuclei of gut epithelial cells, respectively. The green signal in D-type motor neurons is absent in the cell nucleus, which may be due to channel bleeding from the strong 
tdTomato signal. Fluorescent and DIC images of animals carrying only Pacox-1.1::nls::gfp::acox-1.1 3'UTR are also shown. The green signal is not seen around the ventral nerve cord where the cell body of GABAergic neurons resides. $\mathrm{V}$, ventral side; $\mathrm{D}$, dorsal side. Boxed regions are magnified in the insets. Scale bar $=100 \mu \mathrm{m}$.

$\boldsymbol{B}$, Percentages of axons that initiated regeneration $24 \mathrm{~h}$ after laser surgery at the young adult stage. The number of axons examined is shown. Error bars indicate $95 \%$ confidence intervals. ${ }^{\star} P<0.05$, as determined by Fisher's exact test. NS, not significant.

Figure 4. ACOX-1.2 is required for axon regeneration.

$\boldsymbol{A}$, Phylogenetic tree depicting the genetic relationships among ACOX enzymes in $C$. elegans and humans. The phylogenetic tree was constructed using MEGAX software for Mac. The scale bar represents the evolutionary distance calculated using the Poisson correction method based on the number of amino acid substitutions per site.

B, Structures of ACOX-1.2, ACOX-1.3, ACOX-1.4, and ACOX-3. Schematic domain diagrams of $C$. elegans ACOX-1.2, ACOX-1.3, ACOX-1.4, and ACOX-3 and human ACOX3 are shown. Additionally, the gk386052 and gk203391 mutation sites are indicated. The regions deleted in tm5192 and km92 are shown as black bars.

C, Percentages of axons that initiated regeneration $24 \mathrm{~h}$ after laser surgery at the young adult stage. The number of axons examined is shown. Error bars indicate $95 \%$ confidence intervals. ${ }^{*} P<0.05$, ${ }^{* * \star} P<0.001$, as determined by Fisher's exact test. NS, not significant.

Figure 5. Effects of ascaroside ascr\#5 on dauer formation and axon 
Shimizu et al., 34

806

807

808

809

810

\section{regeneration.}

$\boldsymbol{A}$, Dauer induction by ascr\#5. Arrows indicate animals entering the dauer stage. Scale bar $=500 \mu \mathrm{m}$.

$\boldsymbol{B}$, Percentages of dauer formation. Error bars indicate $95 \%$ confidence intervals. ${ }^{\star * \star} P<0.001$, as determined by Fisher's exact test. NS, not significant.

C, Representative D-type motor neurons in animals $24 \mathrm{~h}$ after laser surgery. In acox-1.2 mutants (when ascr\#5 was present), severed axons exhibited regenerated growth cones (yellow arrowheads). In acox-1.2 (when ascr\#5 was absent) and srg-36 srg-37 mutants (when ascr\#5 was present), the proximal ends of axons failed to regenerate (red arrowheads). Scale bar $=10 \mu \mathrm{m}$. $D$, Percentages of axons that initiated regeneration $24 \mathrm{~h}$ after laser surgery at the young adult stage. The number of axons examined is shown. Error bars indicate $95 \%$ confidence intervals. ${ }^{* \star} P<0.01$, as determined by Fisher's exact test. NS, not significant.

\section{Figure 6. The effect of acox-1.2 expression in touch neurons on the} regeneration of the D-type motor axon.

$\boldsymbol{A}$, Schematic representation of motor and touch neurons. The D-type motor neurons (magenta) have cell bodies on the ventral side and extend axonal commissures dorsally. The touch neuron (gray) extends a long axon parallel to the long body axis and crosses almost perpendicular to the axons of D-type motor neurons.

$\boldsymbol{B}$, Percentages of D-type motor axons that initiated regeneration $24 \mathrm{~h}$ after laser surgery in the young adult stage. A schematic representation of motor and touch neurons is shown in the left part. Blue indicates the touch neuron of acox-1.2 mutants expressing the acox-1.2 gene. The number of axons examined is shown. Error bars indicate $95 \%$ confidence intervals. ${ }^{* \star} P<0.01$, as determined 
by Fisher's exact test. NS, not significant.

Figure 7. SRG-36 and SRG-37 are involved in axon regeneration.

$\boldsymbol{A}$, Genomic region surrounding srg-36 and srg-37, deletion breakpoints in the kyIR95 allele, and bicistronic fusion genes. Diagrams for Psrg-36::srg-36::sl2::gfp, Psrg-37::srg-37::s/2::gfp, Punc-25:: srg-36::s/2::gfp, and Punc-25:: srg-37::s/2::gfp are shown in the lower part.

$\boldsymbol{B}-\boldsymbol{D}$, Percentages of axons that initiated regeneration $24 \mathrm{~h}$ after laser surgery at the young adult (YA) stage. The number of axons examined is shown. Error bars indicate $95 \%$ confidence intervals. ${ }^{*} P<0.05,{ }^{* \star} P<0.01$, as determined by Fisher's exact test. NS, not significant.

E, Percentages of axons that initiated regeneration $24 \mathrm{~h}$ after laser surgery at the L4 stage. The number of axons examined is shown. Error bars indicate 95\% confidence intervals. NS, not significant.

\section{Figure 8. Expression pattern of the Psrg-36::srg-36::s/2::gfp gene.}

Fluorescent and DIC images of animals carrying Psrg-36::srg-36::s/2::gfp and Punc-25::tdTomato $1 \mathrm{~h}$ after excision are shown. D-type motor neurons are visualized using tdTomato fused to a nuclear export signal (NES) under control of the unc-25 promoter. Blue, yellow, and white arrowheads indicate a severed axon, the corresponding cell body of the injured neuron, and the head neuron ASI, respectively. V, ventral side; $D$, dorsal side. Boxed regions are shown magnified in the insets. Scale bar $=100 \mu \mathrm{m}$.

Figure 9. A schematic model for regulating axon regeneration by chemical signaling pathways.

Ascr\#5 activates the EGL-30-EGL-8-TPA-1 signaling cascade via 


\begin{abstract}
860 SRG-36/SRG-37 GPCRs to promote axon regeneration. AEA activates the Goo 861 protein GOA-1 via NPR-19/NPR-32 GPCRs, which antagonizes EGL-30, 862 inhibiting axon regeneration. Axon regeneration in $C$. elegans is determined by 863 the balance of stimulatory (ascr\#5) and inhibitory (AEA) chemical signals 864 transduced by $\mathrm{G} \alpha$ protein signaling pathways.
\end{abstract}


Table 1. Strains used in this study.

\begin{tabular}{|c|c|}
\hline Strain & Genotype \\
\hline KU92 & acox-1.4(km92) l; juls76 II \\
\hline KU501 & juls76 II \\
\hline KU456 & egl-30(ad805) l; juls76 II \\
\hline KU457 & egl-30(tg26) I; juls76 II \\
\hline KU1549 & juls76 II; srx-16(tm7585) V \\
\hline KU1550 & acox-1.1(ok2257) I; juls76 II \\
\hline KU1551 & acox-1.1(ok2257) I; juls76 II; kmEx1551[Pacox-1.1::acox-1.1 + Pmyo-2::dsRedmonomer] \\
\hline KU1552 & acox-1.1(ok2257) I; juls76 II; kmEx1552[Pges-1::acox-1.1 + Pmyo-2::dsRedmonomer] \\
\hline KU1553 & acox-1.1(ok2257) I; juls76 II; kmEx1553[Punc-25::acox-1.1 + Pmyo-2::dsRedmonomer] \\
\hline KU1554 & acox-1.1(ok2257) I; juls76 II; kmEx1554[Pmec-7::acox-1.1 + Pmyo-2::dsRedmonomer] \\
\hline KU1555 & acox-1.2(gk386052) l; juls76 II \\
\hline KU1556 & acox-1.2(gk386052) I; juls76 II; kmEx1556[Punc-25::acox-1.2 + Pmyo-2::dsRedmonomer] \\
\hline KU1557 & acox-1.2(gk386052) I; juls76 II; kmEx1557[Pmec-7::acox-1.2 + Pmec-7::gfp + Pmyo-2::dsRedmonomer] \\
\hline KU1558 & acox-1.3(tm5192) I; juls76 II \\
\hline
\end{tabular}









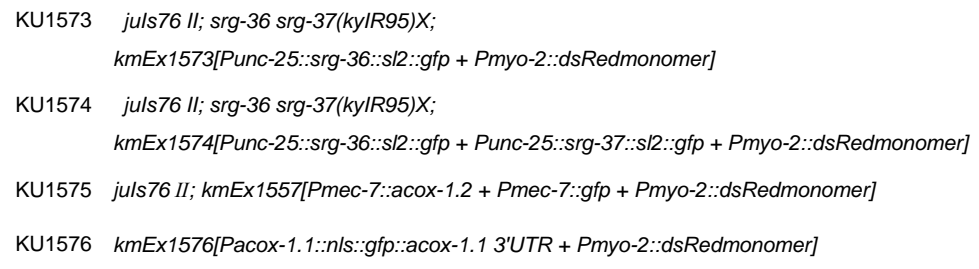


Table 2. Raw data for genotypes tested by axotomy.

\begin{tabular}{|c|c|c|c|c|c|c|c|}
\hline Strain & Genotype (juls76 background) & Stage & $\begin{array}{c}\text { No. of } \\
\text { animals }\end{array}$ & $\begin{array}{l}\text { No. of } \\
\text { axons }\end{array}$ & $\begin{array}{c}\text { No. of } \\
\text { regenerations } \\
\text { (\% of total) }\end{array}$ & $P$-value & $\begin{array}{c}\text { Compared } \\
\text { with }\end{array}$ \\
\hline $\mathrm{KU} 501^{\mathrm{a}}$ & wild type & YA & 24 & 68 & $42(62 \%)$ & - & - \\
\hline KU1549 & $s r x-16(t m 7585)$ & YA & 19 & 53 & $32(60 \%)$ & 0.8493 & $\mathrm{KU} 501^{\mathrm{a}}$ \\
\hline $\mathrm{KU} 501^{\mathrm{b}}$ & wild type & YA & 24 & 64 & $43(67 \%)$ & - & - \\
\hline KU1550 & acox-1.1(ok2257) & YA & 32 & 53 & $22(42 \%)$ & 0.0086 & $\mathrm{KU} 501^{\mathrm{b}}$ \\
\hline KU1551 & acox-1.1(ok2257); Ex[Pacox-1.1::acox-1.1] & YA & 23 & 52 & $38(73 \%)$ & 0.0015 & KU1550 \\
\hline KU456 & egl-30(If) & YA & 18 & 50 & $20(40 \%)^{*}$ & - & - \\
\hline KU1571 & acox-1.1(ok2257); egl-30(If) & YA & 23 & 56 & $27(48 \%)$ & 0.5645 & KU1550 \\
\hline KU457 & egl-30(gf) & YA & 13 & 30 & $21(70 \%)^{*}$ & - & - \\
\hline KU1572 & acox-1.1(ok2257); egl-30(gf) & YA & 33 & 50 & $34(68 \%)$ & 0.0099 & KU1550 \\
\hline KU1561 & daf-22(ok693) & YA & 29 & 50 & $22(44 \%)$ & 0.0217 & $\mathrm{KU} 501^{\mathrm{b}}$ \\
\hline KU1552 & acox-1.1(ok2257); Ex[Pges-1::acox-1.1] & YA & 36 & 52 & $27(52 \%)$ & 0.3307 & KU1550 \\
\hline KU1553 & acox-1.1(ok2257); Ex[Punc-25::acox-1.1] & YA & 35 & 51 & $33(65 \%)$ & 0.0200 & KU1550 \\
\hline KU1554 & acox-1.1(ok2257); Ex[Pmec-7::acox-1.1] & YA & 19 & 51 & $18(35 \%)$ & 0.6863 & KU1550 \\
\hline KU1562 & daf-22(ok693); Ex[Punc-25::daf-22] & YA & 20 & 52 & $34(65 \%)$ & 0.0461 & KU1561 \\
\hline KU1555 ${ }^{\circ}$ & acox-1.2(gk386052) & YA & 35 & 51 & 18 (35\%) & 0.0008 & KU501 ${ }^{\mathrm{b}}$ \\
\hline
\end{tabular}




\begin{tabular}{|c|c|c|c|c|c|c|c|}
\hline KU1556 & acox-1.2(gk386052); Ex[Punc-25::acox-1.2] & YA & 22 & 50 & $30(60 \%)$ & 0.017 & KU1555 \\
\hline KU1558 & acox-1.3(tm5192) & YA & 35 & 52 & $27(52 \%)$ & 0.1268 & $\mathrm{KU} 501^{\mathrm{b}}$ \\
\hline KU1559 & acox-3(gk203391) & YA & 32 & 50 & $28(56 \%)$ & 0.2470 & $\mathrm{KU} 501^{\mathrm{b}}$ \\
\hline KU92 & acox-1.4(km92) & YA & 21 & 53 & $34(59 \%)$ & 1 & $\mathrm{KU} 501^{\mathrm{b}}$ \\
\hline KU1557 & $\operatorname{acox-1.2(gk386052);}$ & YA & 46 & 46 & $14(30 \%)$ & 0.669 & $\mathrm{KU} 1555^{\circ}$ \\
\hline & Ex[Pmec-7::acox-1.2 + Pmec-7::gfp] only D cut & & & & & & \\
\hline \multirow[t]{2}{*}{ KU1557 } & $\operatorname{acox-1.2(gk386052);}$ & YA & 46 & 46 & $30(65 \%)$ & 0.0044 & $\mathrm{KU} 1555^{\circ}$ \\
\hline & Ex[Pmec-7::acox-1.2 + Pmec-7::gfp] PLM+D cut & & & & & & \\
\hline KU1575 & Ex[Pmec-7::acox-1.2 + Pmec-7::gfp] only D cut & YA & 50 & 50 & $35(70 \%)$ & 0.4349 & $\mathrm{KU} 501^{\mathrm{b}}$ \\
\hline KU1575 & Ex[Pmec-7::acox-1.2+Pmec-7::gfp] PLM+D cut & YA & 41 & 41 & $25(61 \%)$ & 1 & $\mathrm{KU} 501^{\mathrm{b}}$ \\
\hline KU1555 ${ }^{d}$ & acox-1.2(gk386052) + EtOH & YA & 15 & 40 & $13(33 \%)$ & - & - \\
\hline KU1555 & $\operatorname{acox}-1.2(g k 386052) \quad+\operatorname{ascr} \# 5(1 \mu \mathrm{M})$ & YA & 19 & 53 & $34(64 \%)$ & 0.0034 & $K U 1555^{d}$ \\
\hline KU1563 & $s r g-36$ srg-37(kylR95) + EtOH & YA & 27 & 68 & $28(41 \%)$ & - & - \\
\hline KU1563 & srg-36 srg-37(kylR95) + ascr\#5 (1 $\mu \mathrm{M})$ & YA & 22 & 60 & $28(47 \%)$ & 0.5939 & KU1563 $3^{\mathrm{e}}$ \\
\hline KU1563 & srg-36 srg-37(kyIR95) & YA & 20 & 50 & $21(42 \%)$ & 0.0083 & $\mathrm{KU} 501^{\mathrm{b}}$ \\
\hline $\mathrm{KU} 456^{9}$ & egl-30(If) & YA & 21 & 54 & $20(37 \%)$ & 0.0016 & $\mathrm{KU} 501^{\mathrm{b}}$ \\
\hline \multirow[t]{2}{*}{ KU1567 } & egl-30(If) srg-36 srg-37(kylR95) & YA & 20 & 50 & $21(42 \%)$ & 1 & KU1563 \\
\hline & & & & & & 0.6892 & $\mathrm{KU} 456^{\mathrm{g}}$ \\
\hline KU1568 & egl-30(gf) srg-36 srg-37(kylR95) & YA & 28 & 62 & $48(77 \%)$ & 0.0001 & KU1563 $3^{f}$ \\
\hline
\end{tabular}




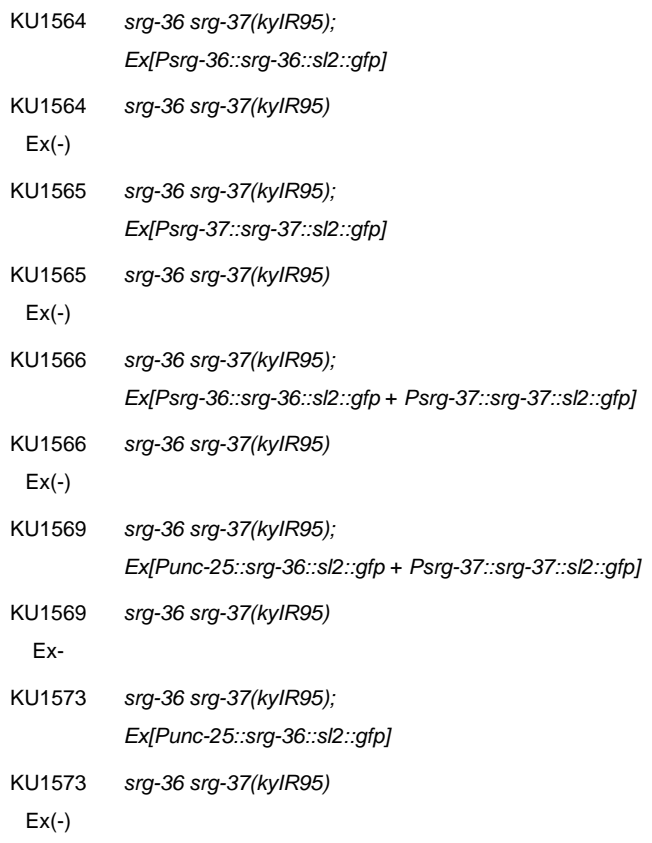

$\begin{array}{llllcc}\text { YA } & 35 & 92 & 45(49 \%) & - & - \\ \text { YA } & 31 & 85 & 31(36 \%) & 0.1283 & \text { KU1564 } \\ \text { YA } & 22 & 63 & 26(41 \%) & - & - \\ \text { YA } & 27 & 72 & 24(33 \%) & 0.3751 & \text { KU1565 } \\ \text { YA } & 25 & 64 & 36(56 \%) & - & - \\ \text { YA } & 29 & 75 & 28(37 \%) & 0.0281 & \text { KU1566 } \\ \text { YA } & 26 & 77 & 42(55 \%) & - & - \\ \text { YA } & 21 & 63 & 22(32 \%) & 0.0281 & \text { KU1569 } \\ & & & & & \\ \text { YA } & 22 & 57 & 19(33 \%) & - & - \\ \text { YA } & 22 & 57 & 19(33 \%) & 1 & \text { KU1573 }\end{array}$




\begin{tabular}{|c|c|c|c|c|c|c|c|}
\hline KU1574 & $\begin{array}{l}\text { srg-36 srg-37(kylR95); } \\
\text { Ex[Punc-25:::srg-36::sl2::gfp + Punc-25::srg-37::s/2::gfp] }\end{array}$ & YA & 30 & 81 & $44(54 \%)$ & - & - \\
\hline $\begin{array}{c}\text { KU1574 } \\
\text { Ex(-) }\end{array}$ & srg-36 srg-37(kyIR95) & YA & 31 & 82 & $27(36 \%)$ & 0.0073 & KU1574 \\
\hline $\mathrm{KU}_{501^{\mathrm{h}}}$ & wild type & L4 & 24 & 54 & $42(78 \%)$ & - & - \\
\hline KU456 & egl-30(If) & L4 & 15 & 41 & $31(76 \%)$ & 0.8112 & KU501 ${ }^{\mathrm{h}}$ \\
\hline KU1563 & srg-36 srg-37(ky/R95) & L4 & 20 & 52 & $39(75 \%)$ & 0.8206 & KU501 ${ }^{\mathrm{h}}$ \\
\hline
\end{tabular}




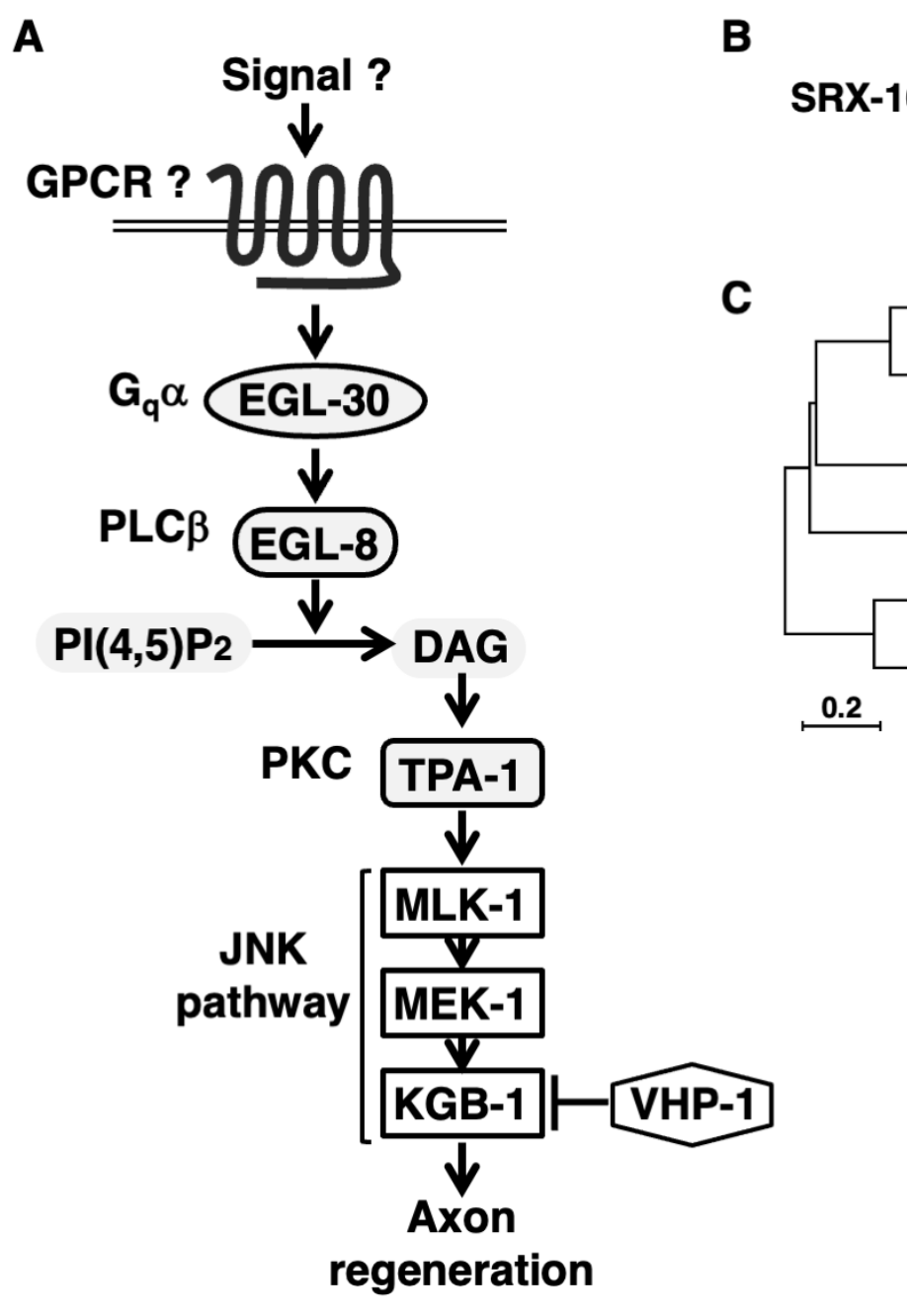

Fig. 1 


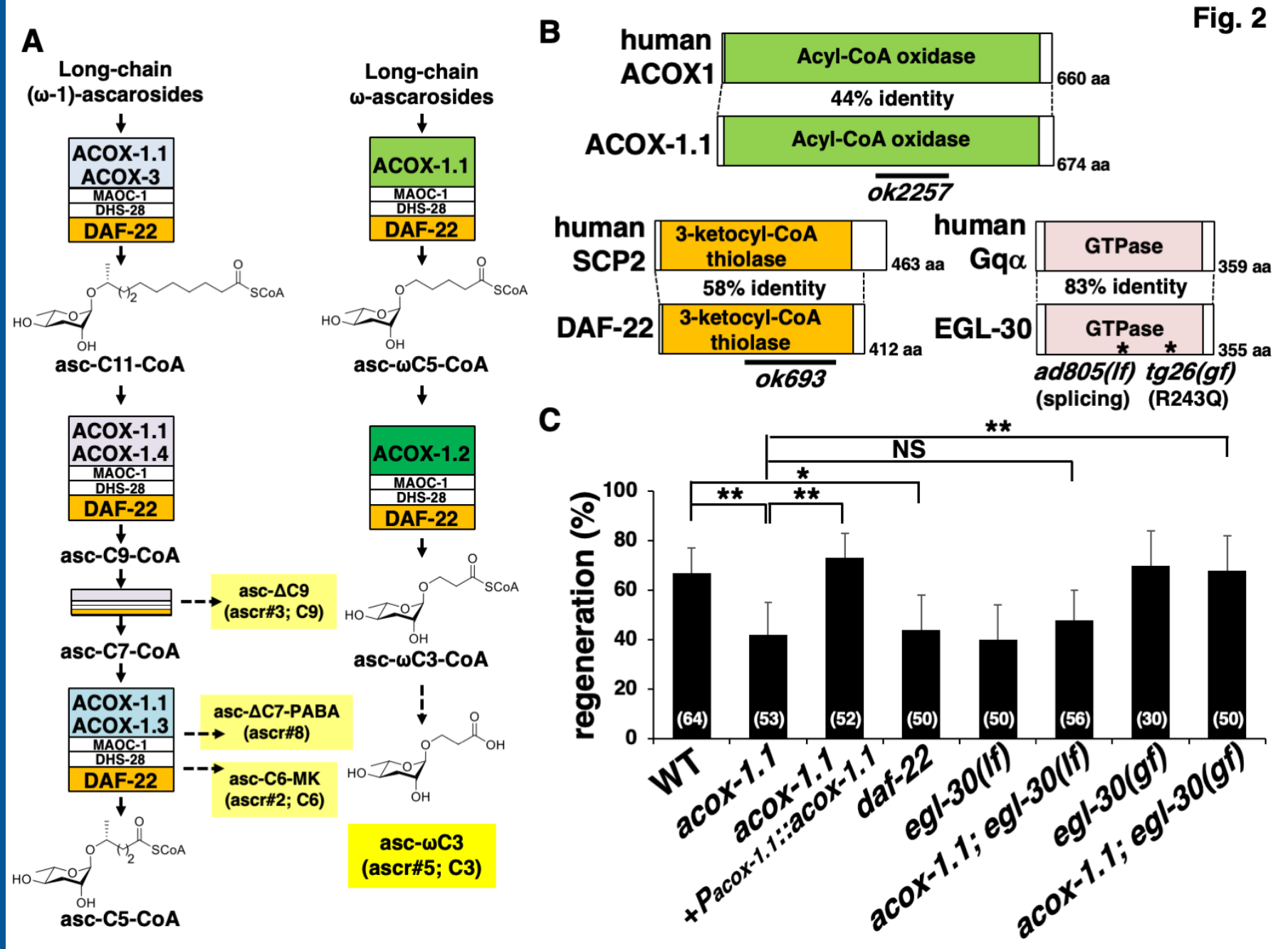


A Pacox-1.1::nls::gfp::

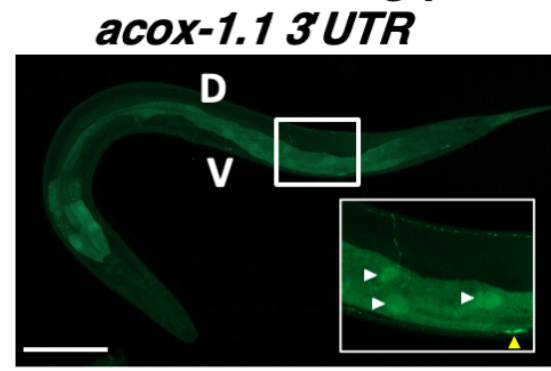

Pacox-1.1::n/s::gfp:: acox-1.1 3UTR

(without marker)

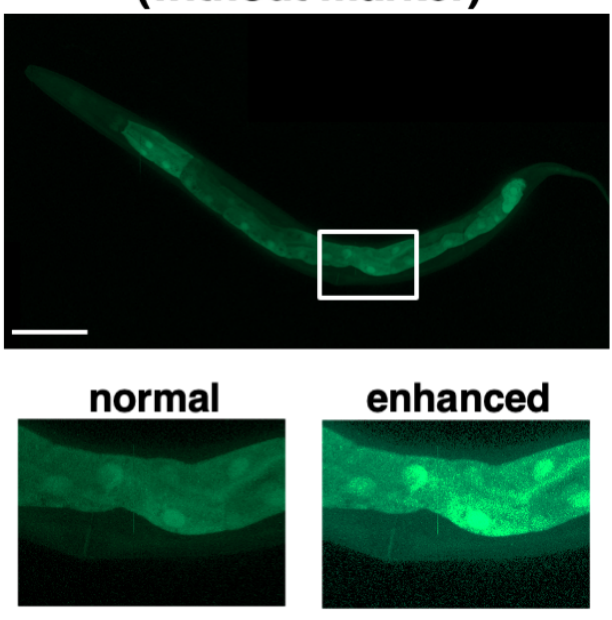

\section{Punc-25::nes::tdTomato}

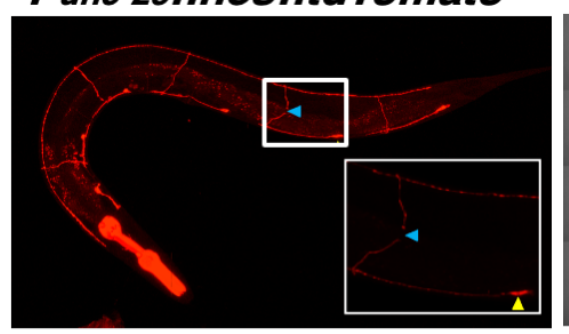

B

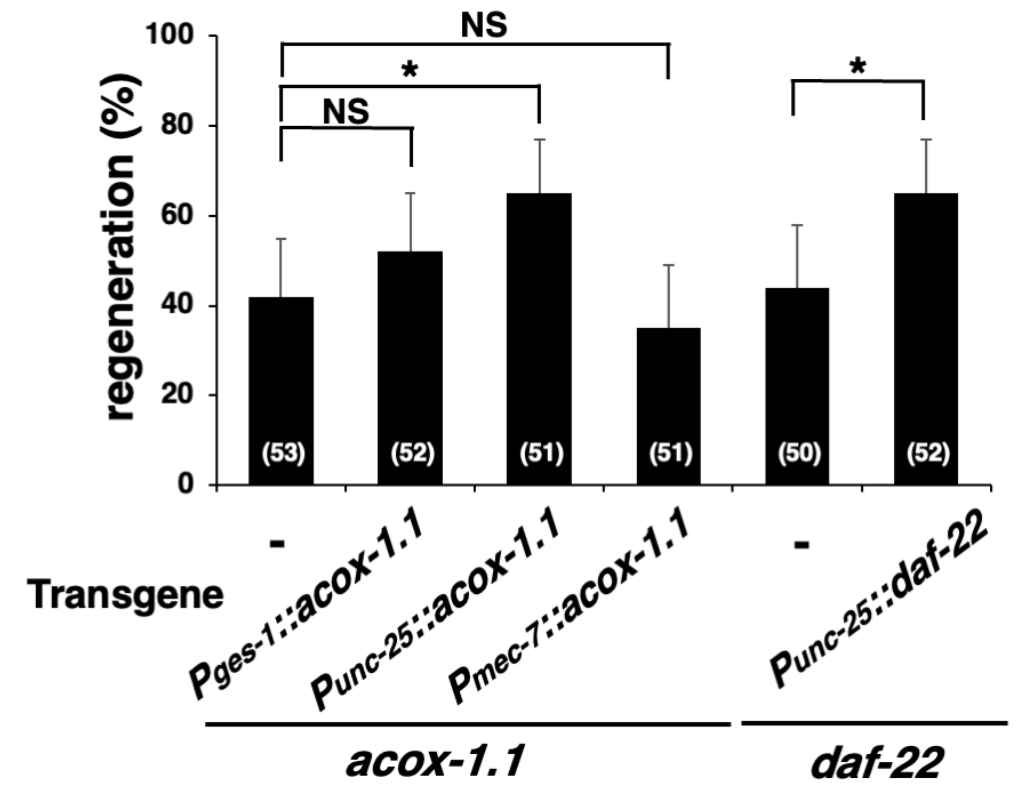

Fig. 3

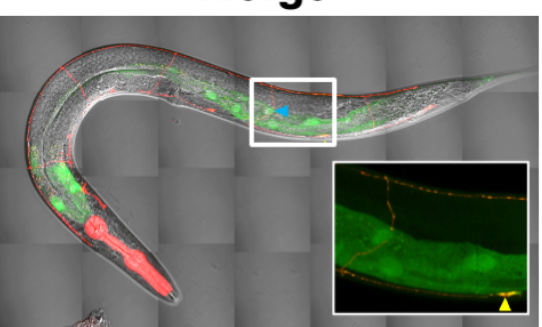


A


Fig. 4
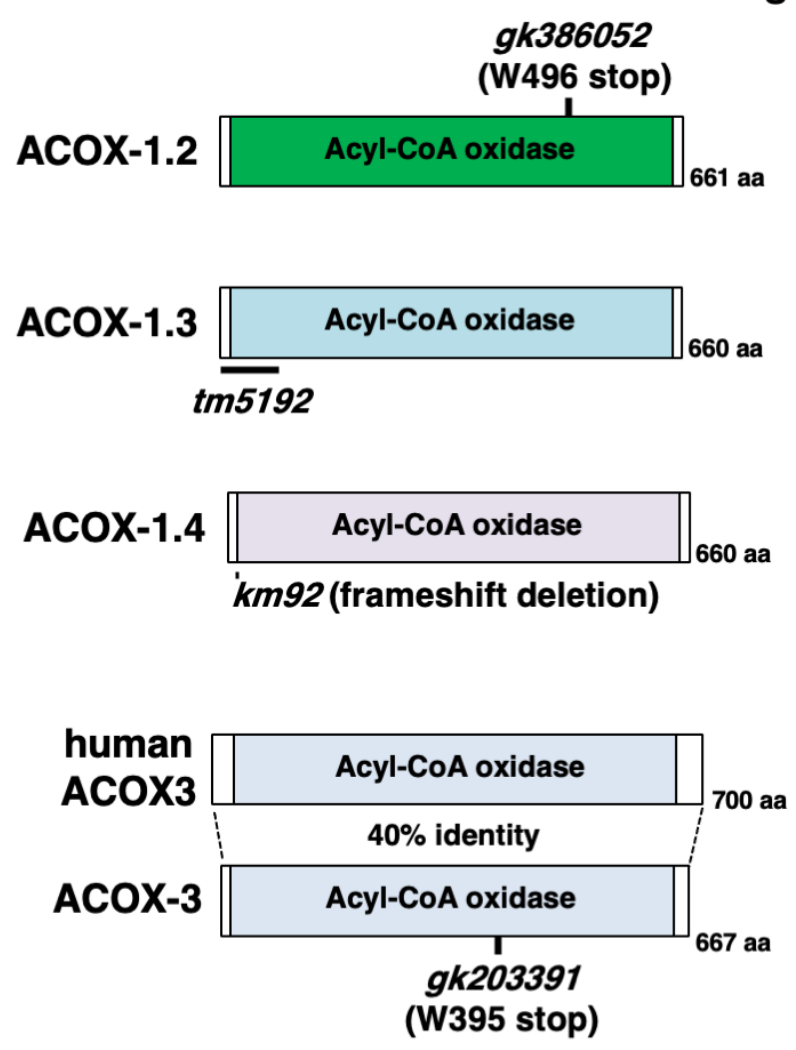
A

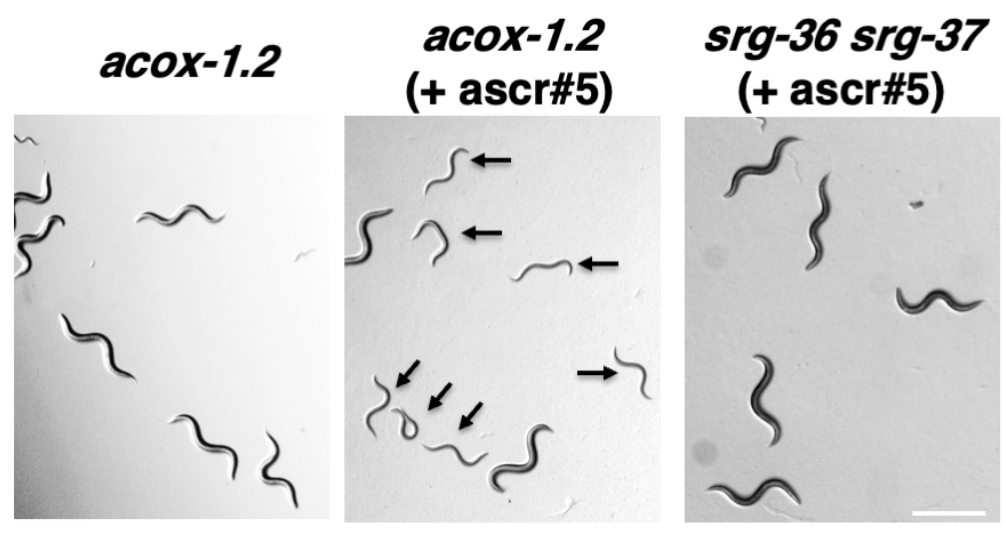

C

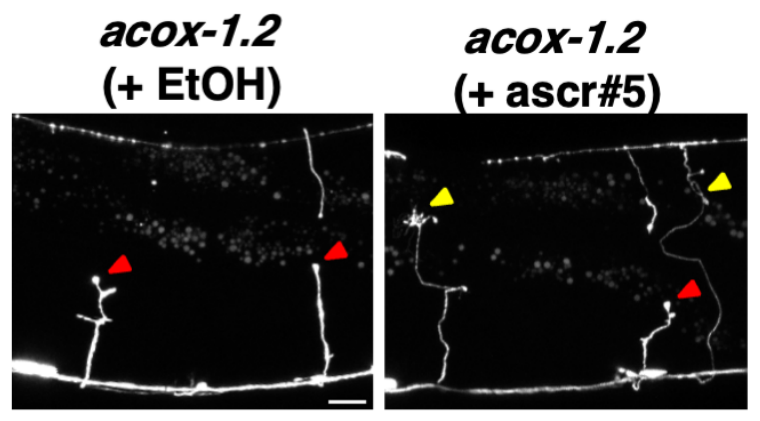

B

Fig. 5

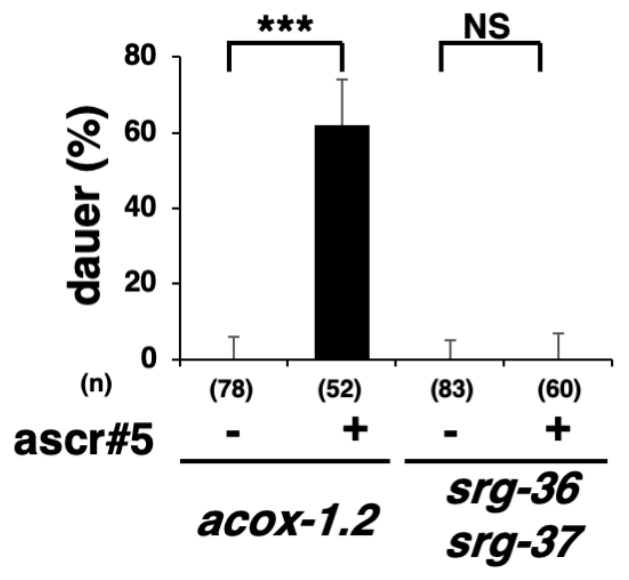

D

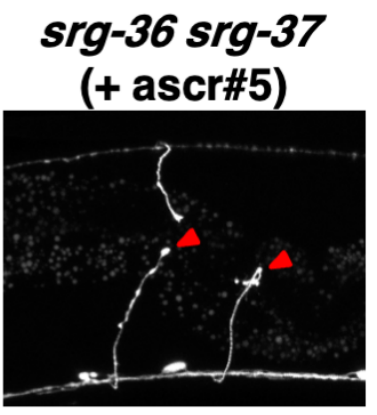

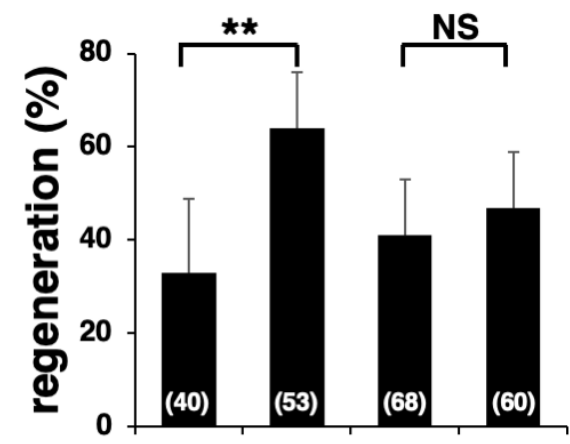

ascr\#5

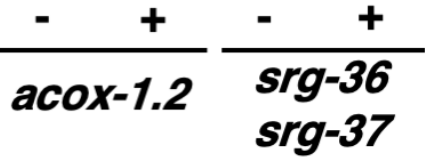


Fig. 6

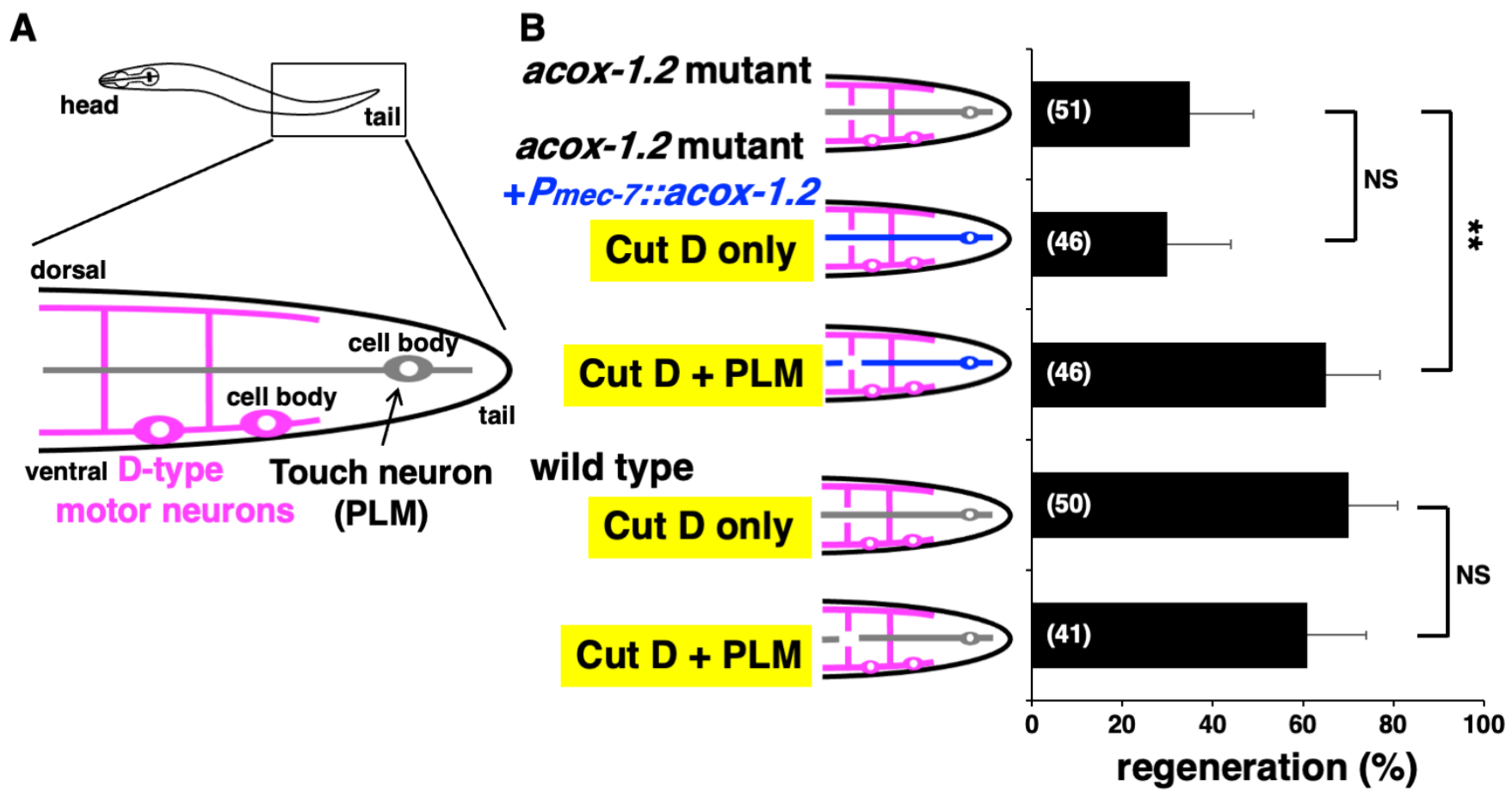


A


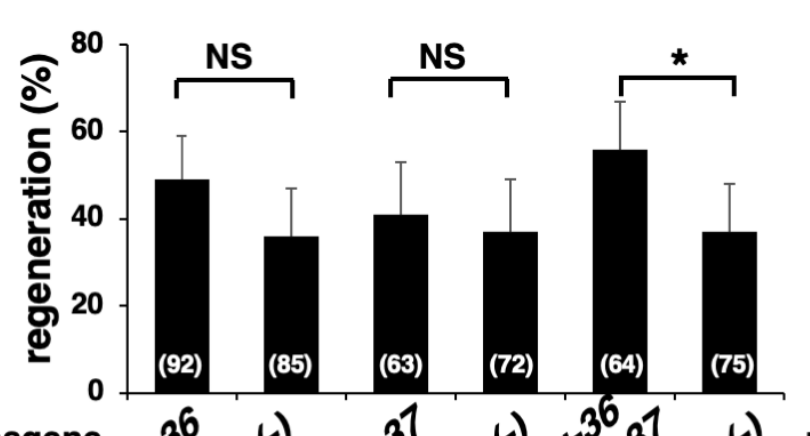

D
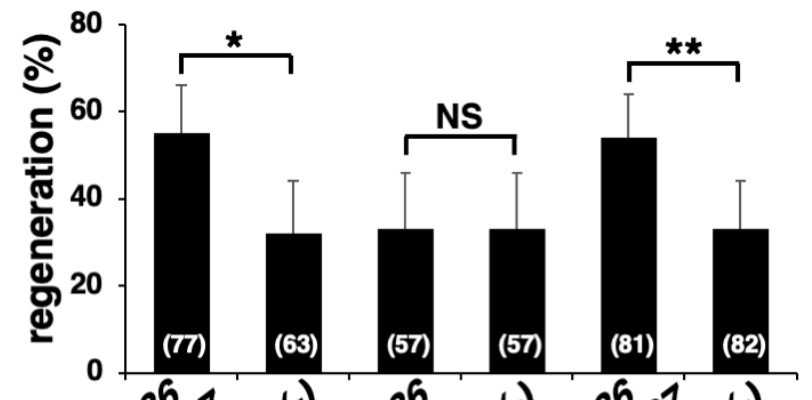
$p \underset{x}{p} p^{129^{25}}$ 
Fig. 8

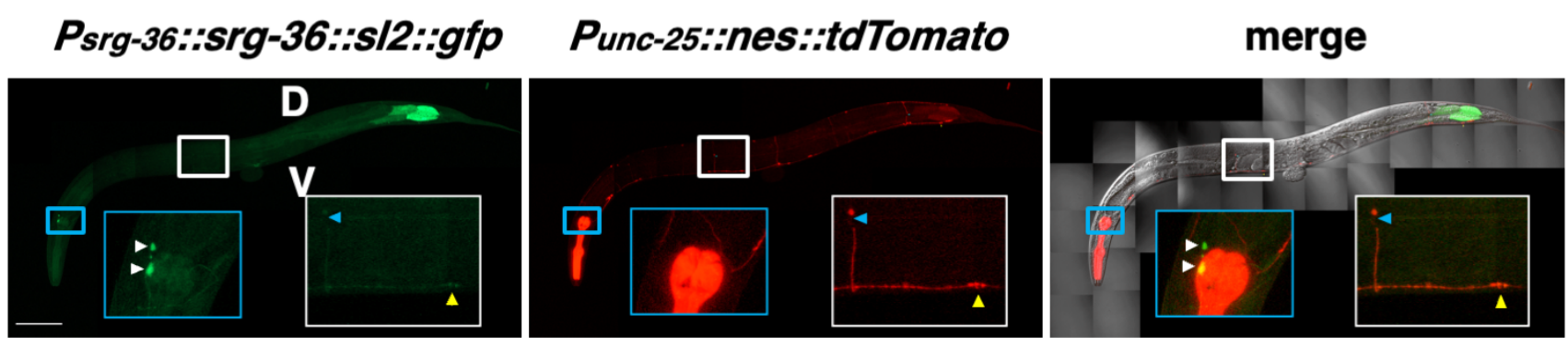


Fig. 9

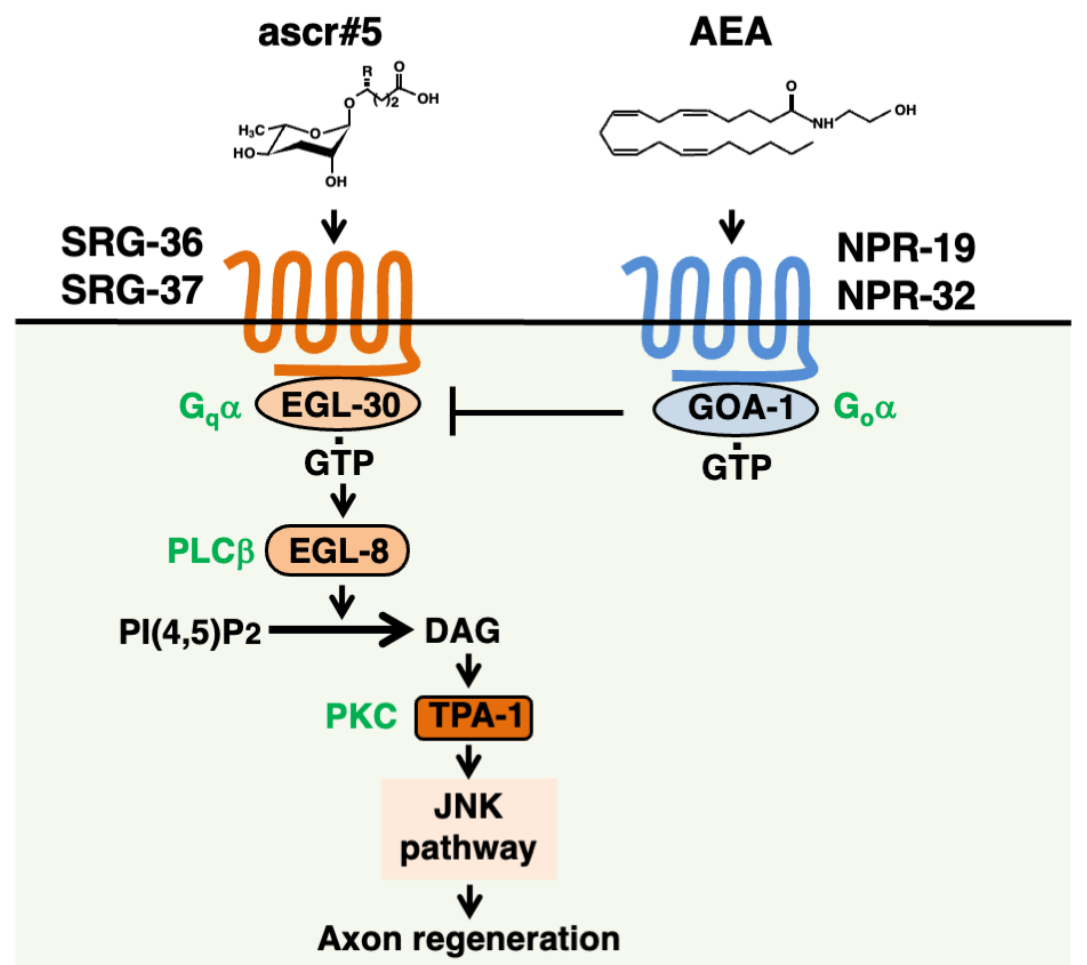

